\title{
Parallel solvers for virtual element discretizations of elliptic equations in mixed form
}

\author{
F. Dassia ${ }^{\mathrm{a}}$, S. Scacchi ${ }^{\mathrm{b}}$ \\ ${ }^{a}$ Dipartimento di Matematica, Università degli Studi di Milano-Bicocca, Via Cozzi, 20133 Milano, Italy \\ ${ }^{b}$ Dipartimento di Matematica, Università degli Studi di Milano, Via Saldini 50, 20133 Milano, Italy
}

\begin{abstract}
The aim of this paper is twofold. On the one hand, we test numerically the performance of mixed virtual elements in three dimensions for the first time in the literature to solve the mixed formulation of three-dimensional elliptic equations on polyhedral meshes. On the other hand, we focus on the parallel solution of the linear system arising from such discretization, considering both direct and iterative parallel solvers. In the latter case, we develop two block preconditioners, one based on the approximate Schur complement and one on a regularization technique. Both these topics are numerically validated by several parallel tests performed on a Linux cluster. More specifically, we show that the proposed VEM discretization recovers the expected theoretical convergence properties and we analize the performance of the direct and iterative parallel solvers taken into account.
\end{abstract}

\section{Introduction}

In recent years, the interest of an increasing number of researchers has focused on the development of numerical methods for the approximation of partial differential equations (PDEs) on polygonal or polyhedral grids, see e.g. [21]. Among the different methodologies, the Virtual Element Method (VEM), introduced in the pioneering paper [15, represents a generalization of the Finite Element Method that can easily handle general polytopal meshes. VEM can be regarded as an evolution of the Mimetic Finite Difference method, see e.g. [22]. So far, VEM has been analyzed for general elliptic problems [20], elasticity [18, 37], Cahn-Hilliard [3], Stokes [23], parabolic and hyperbolic equations [49, 48], discrete fracture networks [24, 36] and several further applications. Different variants of the VEM have been proposed and analysed: $\mathrm{H}(\mathrm{div})$ and $\mathrm{H}(\mathrm{curl})$-conforming [19], serendipity [17] and nonconforming [9, 32] VEM.

VEM for mixed formulation of two-dimensional elliptic problems has been developed in [29, 20]; for alternative polyhedral discretizations of elliptic equations in mixed form see [30]. We recall that second or fourth order elliptic problems can be reformulated as a coupled system by introducing a new variable, typically the gradient of the potential function. This mixed formulation yields a higher order of accuracy for the new variable and has also the favorable property of local mass conservation. However, similarly to other saddle point problems, the resulting linear systems are highly ill-conditioned due to some coefficients, such as a diffusion coefficient, taking widely varying values in the domain where the PDE is posed. Consequently, the solution of saddle point matrix equations with iterative methods (see e.g. [46, 51]) requires the construction of robust and effective preconditioners. Successful preconditioners are based on approximate block factorization, see e.g. [7, 38, 47, 25, 40, 8]. Several Domain Decomposition preconditioners have also been developed for finite element discretizations of such problems, see [39, 42, 43, 44, 50].

To our knowledge, in the VEM literature only a few studies have focused on the conditioning of the stiffness matrix resulting from VEM discretizations (see [41, 34]) and on the development of preconditioners for VEM approximations of PDEs (see [26, 4, 31]). Preconditioners for other

Email addresses: franco.dassi@unimib.it (F. Dassi), simone.scacchi@unimi.it (S. Scacchi) 
polyhedral discretizations have been studied in [6, 5]. We remark that all these works concern scalar elliptic equations in primal form.

The aim of the present contribution is twofold. First, we numerically verify for the first time that the convergence of mixed VEM scheme is in agreement with the expected theoretical estimates. Then, we develop a parallel solver for the solution of the linear systems arising from the discretization process. In the design of the parallel solver, we consider two block preconditioners, one based on the approximate Schur complement and one on a regularization technique, see e.g. [25] and [8], respectively. We compare the iterative methods against the parallel direct solver Mumps [1, 2] by performing several parallel tests on a Linux cluster with varying number of processors, order of VEM discretization and type of polyhedral grid.

The remand of the paper is organized as follows. In Section 2 we give the basic notation and give some useful results on polynomial spaces and decompositions. Then, in Section 3, we briefly introduce the variational formulation of the model problem. In Section 4 we provide the Virtual Element approximation of such problem and some numerical results on the convergence of the proposed schemes. Finally, in Section 5 we focus on the parallel implementation of the discretization process, we describe the block preconditioners used for the solution of the linear system and we show some numerical experiments on a Linux cluster.

\section{Polynomial spaces and bases}

In order to develop the VEM approximation of the $3 \mathrm{~d}$ elliptic equations in mixed form, we need to define suitable basis functions and a polynomial decomposition for certain polynomial spaces introduced below.

Let $\mathbb{P}_{k}(\mathcal{D})$ be the space of polynomials of degree $k$ defined in a general domain $\mathcal{D}$. There are several choices of basis of $\mathbb{P}_{k}(\mathcal{D})$. In the virtual element framework it is convenient to consider the socalled scaled monomial basis [13. Defining the multi-index $\boldsymbol{\alpha}:=\left(\alpha_{1}, \alpha_{2}, \alpha_{3}\right)$ with the usual notation $|\boldsymbol{\alpha}|:=\alpha_{1}+\alpha_{2}+\alpha_{3}$, then a generic scaled monomial on a polyhedron $P$ is

$$
m_{\boldsymbol{\alpha}}:=\left(\frac{\boldsymbol{x}-\boldsymbol{x}_{P}}{h_{P}}\right)^{\boldsymbol{\alpha}}=\left(\frac{x-x_{P}}{h_{P}}\right)^{\alpha_{1}}\left(\frac{y-y_{P}}{h_{P}}\right)^{\alpha_{2}}\left(\frac{z-z_{P}}{h_{P}}\right)^{\alpha_{3}},
$$

where $\boldsymbol{x}_{P}$ is the barycenter of $P$ whose coordinates are $\left(x_{P}, y_{P}, z_{P}\right)$ and $h_{P}$ is the diameter of $P$. It is easy to show that the set of scaled monomials

$$
\mathcal{M}_{k}(P):=\left\{m_{\boldsymbol{\alpha}}: 0 \leq|\boldsymbol{\alpha}| \leq k\right\},
$$

is a basis for $\mathbb{P}_{k}(P)$.

Since we are going to exploit polynomials defined on a face $f$ of $P$, it will be useful to introduce $2 \mathrm{~d}$ scaled monomials. In such case we have to consider a multi-index $\boldsymbol{\beta}:=\left(\beta_{1}, \beta_{2}\right)$ composed by only two components. Then, a generic scaled monomial on a face $f$ is

$$
m_{\boldsymbol{\beta}}^{f}:=\left(\frac{\widetilde{\boldsymbol{x}}-\widetilde{\boldsymbol{x}}_{f}}{h_{f}}\right)^{\boldsymbol{\beta}}=\left(\frac{\widetilde{x}-\widetilde{x}_{f}}{h_{f}}\right)^{\beta_{1}}\left(\frac{\widetilde{y}-\widetilde{y}_{f}}{h_{f}}\right)^{\beta_{2}}
$$

where $\widetilde{\boldsymbol{x}}_{f}=\left(\widetilde{x}_{f}, \widetilde{y}_{f}\right)$ is the barycenter of the face $f$ written in the face local coordinates system $\widetilde{x} O \widetilde{y}$ and $h_{f}$ is the diameter of the face $f$. As in the three dimensional case, the set

$$
\mathcal{M}_{k}(f):=\left\{m_{\boldsymbol{\beta}}^{f}: 0 \leq|\boldsymbol{\beta}| \leq k\right\},
$$

is a basis for $\mathbb{P}_{k}(f)$.

Starting from such polynomial basis, it is possible to define also a vectorial monomial basis for the polynomial space $\left[\mathbb{P}_{k}(\mathcal{D})\right]^{d}$. We refer to this basis as $\left[\mathcal{M}_{k}(P)\right]^{3}$ and $\left[\mathcal{M}_{k}(f)\right]^{2}$ for the three and two dimensional case, respectively. 


\subsection{Polynomial decomposition}

In this subsection we introduce a polynomial decomposition exploited to build the projection operator and the discrete forms of the model problem, see Subsections 4.1 and 4.3 . Let us consider a polyhedron $P$, the vectorial polynomial space $\left[\mathbb{P}_{k}(P)\right]^{3}$ can be split in a direct sum of two spaces

$$
\left[\mathbb{P}_{k}(P)\right]^{3}=\mathcal{G}_{k}(P) \oplus \mathcal{G}_{k}^{\oplus}(P) .
$$

where

$$
\mathcal{G}_{k}(P):=\left\{\boldsymbol{p}_{k} \in\left[\mathbb{P}_{k}(P)\right]^{3}: \exists p_{k+1} \in \mathbb{P}_{k+1}(P) \text { such that } \boldsymbol{p}_{k}=\nabla p_{k+1}\right\},
$$

and $\mathcal{G}_{k}^{\oplus}(P)$ is complement orthogonal to $\mathcal{G}_{k}(P)$ in $\left[\mathbb{P}_{k}(P)\right]^{3}$. A direct consequence of Equation (2) is that a generic vectorial polynomial $\boldsymbol{p}_{k} \in\left[\mathbb{P}_{k}(P)\right]^{3}$ can be written as

$$
\boldsymbol{p}_{k}=\nabla q_{k+1}+\boldsymbol{x} \wedge \boldsymbol{q}_{k-1},
$$

where $q_{k+1} \in \mathbb{P}_{k+1}(P), \boldsymbol{q}_{k-1} \in\left[\mathbb{P}_{k-1}(P)\right]^{3}$ and $\boldsymbol{x}:=(x, y, z)^{T}$ and we further underline that $\nabla q_{k+1} \in \mathcal{G}_{k}(P)$ and $\boldsymbol{x} \wedge \boldsymbol{q}_{k-1} \in \mathcal{G}_{k}^{\oplus}(P)[16]$.

Finding $q_{k+1}$ and $\boldsymbol{q}_{k-1}$ in Equation (3) is not an easy task. However, if we are dealing with vectorial scaled monomials, we found a straightforward recipe to get such decomposition. From now on we will consider a multi-index $\boldsymbol{\alpha}=\left(\alpha_{1}, \alpha_{2}, \alpha_{3}\right)$ and we define the scaled vectorial monomial

$$
\mathbf{m}_{\mathbf{I}}:=\left(\frac{x-x_{P}}{h_{P}}, \frac{y-y_{P}}{h_{P}}, \frac{z-z_{P}}{h_{P}}\right)^{T} .
$$

Proposition 2.1. Considering a vectorial scaled monomial with only the first component different from 0, Equation (3) becomes

$$
\left(\begin{array}{c}
m_{\boldsymbol{\alpha}} \\
0 \\
0
\end{array}\right)=\left(\frac{h_{P}}{|\boldsymbol{\alpha}|+1}\right) \nabla m_{\boldsymbol{\beta}}-\left(\frac{\alpha_{3}}{|\boldsymbol{\alpha}|+1}\right) \mathbf{m}_{\mathbf{I}} \wedge\left(\begin{array}{c}
0 \\
m_{\boldsymbol{\gamma}} \\
0
\end{array}\right)+\left(\frac{\alpha_{2}}{|\boldsymbol{\alpha}|+1}\right) \mathbf{m}_{\mathbf{I}} \wedge\left(\begin{array}{c}
0 \\
0 \\
m_{\boldsymbol{\delta}}
\end{array}\right)
$$

where

$$
\boldsymbol{\beta}=\left(\alpha_{1}+1, \alpha_{2}, \alpha_{3}\right), \quad \gamma=\left(\alpha_{1}, \alpha_{2}, \alpha_{3}-1\right), \quad \text { and } \quad \boldsymbol{\delta}=\left(\alpha_{1}, \alpha_{2}-1, \alpha_{3}\right) .
$$

Proof. Let us compute the gradient of $m_{\boldsymbol{\beta}}$

$$
\nabla m_{\boldsymbol{\beta}}=\frac{1}{h_{P}}\left(\begin{array}{r}
\left(\alpha_{1}+1\right) m_{\boldsymbol{\alpha}} \\
\alpha_{2} m_{\boldsymbol{\delta}_{1}} \\
\alpha_{3} m_{\boldsymbol{\gamma}_{1}}
\end{array}\right)
$$

and the cross products

$$
\mathbf{m}_{\mathbf{I}} \wedge\left(\begin{array}{c}
0 \\
m_{\boldsymbol{\gamma}} \\
0
\end{array}\right)=\left(\begin{array}{c}
-m_{\boldsymbol{\alpha}} \\
0 \\
m_{\boldsymbol{\gamma}_{1}}
\end{array}\right) \quad \text { and } \quad \mathbf{m}_{\mathbf{I}} \wedge\left(\begin{array}{c}
0 \\
0 \\
m_{\boldsymbol{\delta}}
\end{array}\right)=\left(\begin{array}{c}
m_{\boldsymbol{\alpha}} \\
-m_{\boldsymbol{\delta}_{1}} \\
0
\end{array}\right)
$$

where we defined the multi-indexes

$$
\gamma_{1}=\left(\alpha_{1}+1, \alpha_{2}, \alpha_{3}-1\right) \quad \text { and } \quad \boldsymbol{\delta}_{1}=\left(\alpha_{1}+1, \alpha_{2}-1, \alpha_{3}\right) .
$$

Then to get Equation (4), we make a linear combination of such polynomial vectors

$$
c_{1} \frac{1}{h_{P}}\left(\begin{array}{r}
\left(\alpha_{1}+1\right) m_{\boldsymbol{\alpha}} \\
\alpha_{2} m_{\boldsymbol{\delta}_{1}} \\
\alpha_{3} m_{\boldsymbol{\gamma}_{1}}
\end{array}\right)+c_{2}\left(\begin{array}{c}
-m_{\boldsymbol{\alpha}} \\
0 \\
m_{\boldsymbol{\gamma}_{1}}
\end{array}\right)+c_{3}\left(\begin{array}{c}
m_{\boldsymbol{\alpha}} \\
-m_{\boldsymbol{\delta}_{1}} \\
0
\end{array}\right) .
$$


We observe that the particular choice of the multi-indexes $\boldsymbol{\beta}, \boldsymbol{\gamma}$ and $\boldsymbol{\delta}$, leads to a linear combination of vectorial monomials which have monomials with the same multi-index on each component. To complete the proof, we solve the following linear system in the variables $c_{1}, c_{2}$ and $c_{3}$

$$
\left\{\begin{array}{rl}
\frac{1}{h_{P}}\left(\alpha_{1}+1\right) c_{1}-c_{2}+c_{3} & =1 \\
\frac{1}{h_{P}} \alpha_{2} c_{1}-c_{3} & =0 \\
\frac{1}{h_{P}} \alpha_{3} c_{1}+c_{2} & =0
\end{array} .\right.
$$

In Propositions 2.2 and 2.3, we provide similar results for vectorial monomials which have the other components different from 0 . We do not show the proofs of such propositions since they are similar to the one of Proposition 2.1.

Proposition 2.2. Considering a vectorial scaled monomial with only the second component different from 0, Equation (3) becomes

$$
\left(\begin{array}{c}
0 \\
m_{\boldsymbol{\alpha}} \\
0
\end{array}\right)=\left(\frac{h_{P}}{|\boldsymbol{\alpha}|+1}\right) \nabla m_{\boldsymbol{\beta}}+\left(\frac{\alpha_{3}}{|\boldsymbol{\alpha}|+1}\right) \mathbf{m}_{\mathbf{I}} \wedge\left(\begin{array}{c}
m_{\boldsymbol{\gamma}} \\
0 \\
0
\end{array}\right)-\left(\frac{\alpha_{1}}{|\boldsymbol{\alpha}|+1}\right) \mathbf{m}_{\mathbf{I}} \wedge\left(\begin{array}{c}
0 \\
0 \\
m_{\boldsymbol{\delta}}
\end{array}\right)
$$

where

$$
\boldsymbol{\beta}=\left(\alpha_{1}, \alpha_{2}+1, \alpha_{3}\right), \quad \gamma=\left(\alpha_{1}, \alpha_{2}, \alpha_{3}-1\right), \quad \text { and } \quad \boldsymbol{\delta}=\left(\alpha_{1}-1, \alpha_{2}, \alpha_{3}\right) .
$$

Proposition 2.3. Considering a vectorial scaled monomial with only the third component different from 0, Equation (3) becomes

$$
\left(\begin{array}{c}
0 \\
0 \\
m_{\boldsymbol{\alpha}}
\end{array}\right)=\left(\frac{h_{P}}{|\boldsymbol{\alpha}|+1}\right) \nabla m_{\boldsymbol{\beta}}+\left(\frac{\alpha_{1}}{|\boldsymbol{\alpha}|+1}\right) \mathbf{m}_{\mathbf{I}} \wedge\left(\begin{array}{c}
0 \\
m_{\boldsymbol{\gamma}} \\
0
\end{array}\right)-\left(\frac{\alpha_{2}}{|\boldsymbol{\alpha}|+1}\right) \mathbf{m}_{\mathbf{I}} \wedge\left(\begin{array}{c}
m_{\boldsymbol{\delta}} \\
0 \\
0
\end{array}\right)
$$

where

$$
\boldsymbol{\beta}=\left(\alpha_{1}, \alpha_{2}, \alpha_{3}+1\right), \quad \gamma=\left(\alpha_{1}-1, \alpha_{2}, \alpha_{3}\right), \quad \text { and } \quad \boldsymbol{\delta}=\left(\alpha_{1}, \alpha_{2}-1, \alpha_{3}\right) .
$$

Remark 2.1. A decomposition of a vectorial scaled monomial with more than one component different from zero can be obtained by summing the decompositions provided by the Propositions 2.1, 2.2 and 2.3.

Remark 2.2. Since the first term of decompositions 2.1. 2.2 and 2.3 are gradients, we are able to generate any vectorial polynomial $\boldsymbol{p}_{k} \in \mathcal{G}_{k}^{\oplus}(P)$ starting from a linear combinations of $\mathbf{m}_{\mathbf{I}} \wedge \mathbf{m}$ where $\mathbf{m} \in\left[\mathcal{M}_{k-1}(P)\right]^{3}$.

\subsection{Basis for $\mathcal{G}_{k}(P)$ and $\mathcal{G}_{k}^{\oplus}(P)$}

The space relation provided in Equation (2) suggests another polynomial vectorial basis for $\left[\mathbb{P}_{k}(P)\right]^{3}$. Indeed, one can think to combine the basis of $\mathcal{G}_{k}(P)$ and $\mathcal{G}_{k}^{\oplus}(P)$ to get a new basis of $\left[\mathbb{P}_{k}(P)\right]^{3}$, i.e.

$$
\boldsymbol{p}_{k}=\sum_{i=1}^{n} c_{i} \boldsymbol{a}_{i}+\sum_{j=1}^{m} d_{i} \boldsymbol{b}_{i}
$$

where $\left\{\boldsymbol{a}_{i}\right\}_{i=1}^{n}$ and $\left\{\boldsymbol{b}_{j}\right\}_{j=1}^{m}$ are two sets of vectorial basis function of $\mathcal{G}_{k}(P)$ and $\mathcal{G}_{k}^{\oplus}(P)$, respectively. 
Basis for $\mathcal{G}_{k}(P)$. Since the operator $\nabla$ is an isomorphism between $\mathbb{P}_{k+1}(P) \backslash \mathbb{R}$ and $\mathcal{G}_{k}(P)$, we know that a basis of $\mathbb{P}_{k+1}(P) \backslash \mathbb{R}$ will be mapped into a basis of $\mathcal{G}_{k}(P)$ by the operator $\nabla$. Consequently, we have

$$
\mathcal{G}_{k}(P)=\operatorname{span}\left\{\nabla m_{\boldsymbol{\alpha}}\right\}
$$

where $0<|\boldsymbol{\alpha}| \leq k+1$.

Basis for $\mathcal{G}_{k}^{\oplus}(P)$. Starting from Equation (3) and Remark 2.2, the idea will be to exploit the basis of $\left[\mathbb{P}_{k-1}(P)\right]^{3}$ to get $\mathrm{s}$ basis for $\mathcal{G}_{k}^{\oplus}(P)$. However, there are some difficulties which require additional observations and resutls.

Proposition 2.4. The linear function $\boldsymbol{x} \wedge *:\left[\mathbb{P}_{k-1}(P)\right]^{3} \rightarrow \mathcal{G}_{k}^{\oplus}(P)$ is not an isomorphism.

Proof. Suppose that $\boldsymbol{x} \wedge *$ is an isomorphism, then $\left[\mathbb{P}_{k-1}(P)\right]^{3}$ and $\mathcal{G}_{k}^{\oplus}(P)$ are isomorph and the following relation holds

$$
\operatorname{dim}\left(\left[\mathbb{P}_{k-1}(P)\right]^{3}\right)=\operatorname{dim}\left(\mathcal{G}_{k}^{\oplus}(P)\right) .
$$

Let us compute the dimensions of these two spaces separately

$$
\operatorname{dim}\left(\left[\mathbb{P}_{k-1}(P)\right]^{3}\right)=\frac{k(k+1)(k+2)}{2}=\frac{k^{3}+4 k^{2}+2 k}{2} .
$$

From Equations (2) and (5) we have

$$
\begin{aligned}
\operatorname{dim}\left(\mathcal{G}_{k}^{\oplus}(P)\right) & =\operatorname{dim}\left(\left[\mathbb{P}_{k}(P)\right]^{3}\right)-\operatorname{dim}\left(\mathcal{G}_{k}(P)\right) \\
& =\frac{(k+1)(k+2)(k+3)}{2}-\left(\frac{(k+1)(k+2)(k+3)}{6}-1\right) \\
& =\frac{2 k^{3}+9 k^{2}+7 k}{6} .
\end{aligned}
$$

Since we do not get the same dimension of the spaces,

$$
\frac{2 k^{3}+9 k^{2}+7 k}{6} \neq \frac{k^{3}+4 k^{2}+2 k}{2}
$$

$\left[\mathbb{P}_{k-1}(P)\right]^{3}$ and $\mathcal{G}_{k}^{\oplus}(P)$ can not be isomorph and consequently $x \wedge *$ can not be an isomorphism.

Corollary 2.1. Since $\boldsymbol{x} \wedge *$ is not an isomorphism, its kernel is not trivial and it is the set

$$
\mathcal{K}_{k-1}(P):=\left\{\boldsymbol{p}_{k-1} \in\left[\mathbb{P}_{k-1}(P)\right]^{3}: \boldsymbol{p}_{k-1}=\left(\begin{array}{c}
x \\
y \\
z
\end{array}\right) p_{k-2}, \forall p_{k-2} \in \mathbb{P}_{k-2}(P)\right\} .
$$

Proof. First of all we check if a generic element of $\mathcal{K}_{k-1}(P)$ is mapped to the null polynomial via the operator $x \wedge *$. Let us consider a generic polynomial $p_{k-2} \in \mathbb{P}_{k-2}(P)$

$$
\left[\left(\begin{array}{l}
x \\
y \\
z
\end{array}\right) \wedge\left(\begin{array}{l}
x \\
y \\
z
\end{array}\right) p_{k-2}\right]=p_{k-2}\left[\left(\begin{array}{l}
x \\
y \\
z
\end{array}\right) \wedge\left(\begin{array}{l}
x \\
y \\
z
\end{array}\right)\right]=0
$$

Now we have to verify that only these elements are mapped to the null polynomial. To achieve this goal, we prove that $\mathcal{K}_{k-1}(P)$ has the same dimension of $\operatorname{ker}(x \wedge *)$. Indeed, the dimension of $\mathcal{K}_{k-1}(P)$ is

$$
\frac{k(k-1)(k+1)}{6},
$$


and that the dimension of the $\operatorname{ker}(x \wedge *)$ is given by the following relation

$$
\operatorname{dim}\left(\left[\mathbb{P}_{k-1}(P)\right]^{3}\right)=\operatorname{dim}\left(\mathcal{G}_{k}^{\oplus}(P)\right)+\operatorname{dim}(\operatorname{ker}(x \wedge *)),
$$

which implies that

$$
\begin{aligned}
\operatorname{dim}(\operatorname{ker}(x \wedge *)) & =\operatorname{dim}\left[\mathbb{P}_{k-1}(P)\right]^{3}-\operatorname{dim}\left(\mathcal{G}_{k}^{\oplus}(P)\right) \\
& =\frac{k^{3}+3 k^{2}+2 k}{2}-\frac{2 k^{3}+9 k^{2}+7 k}{6} \\
& =\frac{k(k-1)(k+1)}{6} .
\end{aligned}
$$

Remark 2.3. Proposition 2.4 still holds if we consider the linear operator $\mathbf{m}_{\mathbf{I}} \wedge *$ instead of $\boldsymbol{x} \wedge *$.

Since $\mathbf{m}_{\mathbf{I}} \wedge *:\left[\mathbb{P}_{k-1}(P)\right]^{3} \rightarrow \mathcal{G}_{k}^{\oplus}(P)$ is not an isomorphism, a basis in the space $\left[\mathbb{P}_{k-1}(P)\right]^{3}$ is not mapped to a basis of $\mathcal{G}_{k}^{\oplus}(P)$ so we can not proceed in a similar way as for finding a basis for $\mathcal{G}_{k}(P)$.

Proposition 2.5. Let us consider the a multi-index $\boldsymbol{\alpha}$, then the following relation holds

$$
\mathbf{m}_{\mathbf{I}} \wedge\left(\begin{array}{c}
m_{\boldsymbol{\alpha}} \\
0 \\
0
\end{array}\right)=-\mathbf{m}_{\mathbf{I}} \wedge\left(\begin{array}{c}
0 \\
m_{\boldsymbol{\beta}} \\
0
\end{array}\right)-\mathbf{m}_{\mathbf{I}} \wedge\left(\begin{array}{c}
0 \\
0 \\
m_{\boldsymbol{\gamma}}
\end{array}\right)
$$

when

$$
\boldsymbol{\beta}=\left(\alpha_{1}-1, \alpha_{2}+1, \alpha_{3}\right) \quad \text { and } \quad \boldsymbol{\gamma}=\left(\alpha_{1}-1, \alpha_{2}, \alpha_{3}+1\right) .
$$

Proof. The proof of this result is a simple computation of cross products.

Proposition 2.5 suggests us that we can replace the vectorial polynomials $\left(m_{\boldsymbol{\alpha}}, 0,0\right)^{T}$ with a linear combination of other two vectorial monomials $\left(0, m_{\boldsymbol{\beta}}, 0\right)^{T}$ and $\left(0,0, m_{\boldsymbol{\gamma}}\right)^{T}$ in the decompositions of Propositions 2.2 and 2.3. Moreover, if we define the set of vectorial monomials

$\mathcal{M}_{k}^{\mathcal{G}, \oplus}(P):=\left\{\left(\begin{array}{c}m_{\boldsymbol{\alpha}} \\ 0 \\ 0\end{array}\right): m_{\boldsymbol{\alpha}} \in \mathcal{N}_{k}(P)\right\} \cup\left\{\left(\begin{array}{c}0 \\ m_{\boldsymbol{\beta}} \\ 0\end{array}\right): m_{\boldsymbol{\beta}} \in \mathcal{M}_{k}(P)\right\} \cup\left\{\left(\begin{array}{c}0 \\ 0 \\ m_{\boldsymbol{\gamma}}\end{array}\right): m_{\boldsymbol{\gamma}} \in \mathcal{M}_{k}(P)\right\}$,

where

$$
\mathcal{N}_{k}(P):=\left\{m_{\boldsymbol{\alpha}}: 0<|\boldsymbol{\alpha}| \leq k, \alpha_{1}=0\right\},
$$

it follows that any vector in $\mathcal{G}_{k}^{\oplus}(P)$ can be written as a linear combination of the elements in $\mathcal{M}_{k-1}^{\mathcal{G}, \oplus}(P)$, i.e. $\forall \boldsymbol{p}_{k} \in \mathcal{G}_{k}^{\oplus}(P)$ we have

$$
\boldsymbol{p}_{k}=c_{0} \mathbf{m}_{\mathbf{I}} \wedge \mathbf{m}_{0}^{\oplus}+c_{1} \mathbf{m}_{\mathbf{I}} \wedge \mathbf{m}_{1}^{\oplus}+\ldots+c_{n} \mathbf{m}_{\mathbf{I}} \wedge \mathbf{m}_{n}^{\oplus},
$$

where $c_{0}, c_{1}, \ldots c_{n} \in \mathbb{R}$ and $\mathbf{m}_{0}^{\oplus}, \mathbf{m}_{1}^{\oplus}, \ldots \mathbf{m}_{n}^{\oplus} \in \mathcal{M}_{k-1}^{\mathcal{G}, \oplus}(P)$.

Now if we show that the dimension of $\mathcal{M}_{k-1}^{\mathcal{G}, \oplus}(P)$ coincides with the dimension of $\mathcal{G}_{k}^{\oplus}(P)$, the image of the set $\mathcal{M}_{k-1}^{G, \oplus}(P)$ via the operator $\mathbf{m}_{\mathbf{I}} \wedge *$ is a basis for $\mathcal{G}_{k}^{\oplus}(P)$.

Proposition 2.6. The following space relation holds

$$
\operatorname{dim}\left(\mathcal{M}_{k-1}^{\mathcal{G}, \oplus}(P)\right)=\operatorname{dim}\left(\mathcal{G}_{k}^{\oplus}(P)\right) .
$$


Proof. We already show in the proof of Proposition 2.4 that

$$
\operatorname{dim}\left(\mathcal{G}_{k}^{\oplus}(P)\right)=\frac{2 k^{3}+9 k^{2}+7 k}{6},
$$

then we have that

$$
\operatorname{dim}\left(\mathcal{M}_{k-1}^{\mathcal{G}, \oplus}(P)\right)=\frac{k(k+1)}{2}+2 \frac{k(k+1)(k+2)}{6}=\frac{2 k^{3}+9 k^{2}+7 k}{6},
$$

and this complete the proof.

\section{Model problem: elliptic equation in mixed form}

The object of this work is the solution of the variational problem arising from the mixed formulation of a scalar elliptic equation in three spatial dimension.

Let $\Omega$ be a bounded Lipschitz domain in $\mathbb{R}^{3}$, whose boundary is denoted by $\partial \Omega$. We define the function spaces

$$
\mathbf{V}:=\left\{\mathbf{u} \in H(\operatorname{div}, \Omega): \mathbf{u} \cdot \mathbf{n}=u_{N} \quad \text { on } \partial \Omega\right\}
$$

and

$$
Q:=\left\{q \in L^{2}(\Omega): \int_{\Omega} q d x=0\right\}
$$

where $H(\operatorname{div}, \Omega)$ is the space of vector-valued functions such that $\mathbf{u}$ and $\operatorname{div}(\mathbf{u})$ belong to $\left[L^{2}(\Omega)\right]^{3}$ and $L^{2}(\Omega)$, respectively, and $u_{N}$ is the given Neumann datum.

The variational problem reads:

$$
\left\{\begin{array}{rlrl}
\text { find }(\mathbf{u}, p) \in(\mathbf{V}, Q): & & \\
\boldsymbol{a}(\mathbf{u}, \mathbf{v})-\boldsymbol{b}(\mathbf{v}, p) & =0 & & \forall \mathbf{v} \in \mathbf{V} \\
\boldsymbol{b}(\mathbf{u}, q) & =\int_{\Omega} f q d x & & \forall q \in Q
\end{array}\right.
$$

where

$$
\begin{aligned}
\boldsymbol{a}(\mathbf{u}, \mathbf{v}) & :=\int_{\Omega} \nu(x) \mathbf{u} \cdot \mathbf{v} d x \\
\boldsymbol{b}(\mathbf{u}, q) & :=\int_{\Omega} \operatorname{div}(\mathbf{u}) q d x
\end{aligned}
$$

$f$ is a given function and $\nu(x)$ is a positive piecewise constant scalar function.

From the applications point of view, problem (11) arises in the context of multiphase incompressible flow through porous media, see e.g. [27. Functions $\mathbf{u}$ and $p$ are usually called velocity and pressure. We refer to [27] for the mathematical analysis of such problem.

\section{Virtual element discretization}

Let $\Omega_{h}$ be a polyhedral decomposition of a three dimensional domain $\Omega$. To solve Problem (11), we follow a standard VEM approach. We define local spaces in a generic polyhedron $P$, then we glue them together to get the global one. Since we are dealing with the mixed formulation of the Laplace problem, we have to consider two types of spaces, one for the velocity $\boldsymbol{V}_{k, h}\left(\Omega_{h}\right)$ and one for the pressure $Q_{k-1, h}\left(\Omega_{h}\right)$. 


\subsection{Definition of $\boldsymbol{V}_{k, h}\left(\Omega_{h}\right)$}

To discretize the velocity, we take the virtual element $3 \mathrm{~d}$ face space introduced in [19]. In this paper we will give a brief description on such local space, we refer to Section 5 of [19] to have a deeper analysis. However, since the definition of the degrees of freedom in [19] is not appropriate from the practical and implementation point of view, we will make a more concrete definition of them.

Given a polyhedron $P$, we define the space

$$
\begin{aligned}
\boldsymbol{V}_{k, h}^{\mathrm{face}}(P):=\left\{\mathbf{v}_{h} \in H(\operatorname{div} ; P) \cap H(\mathbf{c u r l}) \quad:\right. & \mathbf{v}_{h} \cdot \mathbf{n}_{f} \in \mathbb{P}_{k}(f) \quad \forall f \in \partial P \\
& \operatorname{div}\left(\mathbf{v}_{h}\right) \in \mathbb{P}_{k-1}(P), \\
& \left.\operatorname{curl}\left(\mathbf{v}_{h}\right) \in\left[\mathbb{P}_{k-1}(P)\right]^{3}\right\} .
\end{aligned}
$$

The degrees of freedom of such space are

- normal face moments

$$
\frac{1}{|f|} \int_{f}\left(\mathbf{v}_{h} \cdot \mathbf{n}_{f}\right) m_{\boldsymbol{\beta}} \mathrm{d} f, \quad \forall f \in \partial P, \quad \forall m_{\boldsymbol{\beta}} \in \mathcal{M}_{k}(f),
$$

where $|f|$ denotes the area of the face $f$;

- internal gradient moments

$$
\frac{h_{P}}{|P|} \int_{P} \mathbf{v}_{h} \cdot \nabla m_{\boldsymbol{\alpha}} \mathrm{d} P, \quad \forall m_{\boldsymbol{\alpha}} \in \mathcal{M}_{k-1}(P) \backslash \mathcal{M}_{0}(P),
$$

where $|P|$ denotes the volume of $P$;

- internal cross moments

$$
\frac{1}{|P|} \int_{P} \mathbf{v}_{h} \cdot\left(\mathbf{m}_{\mathbf{I}} \wedge \mathbf{m}\right) \mathrm{d} P, \quad \forall \mathbf{m} \in \mathcal{M}_{k-1}^{\mathcal{G}, \oplus}(P) .
$$

In Equations (14), (15) and (16), we highlight the scaling factors. In a virtual element framework the degrees of freedom have to scale as 1 to get a better conditioning of the stiffness matrix [13]. Moreover, we explicitly show which polynomials are taken to define such degrees of freedom. Since we have to consider a set of linearly independent conditions, we use the basis functions provided in Subsection 2.2. We will see that such choice makes computations easier and more straightforward with respect to the other ones. This fact will become clearer when we show how to compute $\operatorname{div}\left(\mathbf{v}_{h}\right)$ and the projection operator $\boldsymbol{\Pi}_{k}^{0}$.

Remark 4.1. The condition on the normal face moments could be replaced by the evaluation of $\mathbf{v}_{h} \cdot \mathbf{n}_{f}$ at suitable points on the face $f$ [19]. However, finding a good position of such points could be not so straightforward when we are dealing with polygons so we use the degrees of freedom in Equation (14).

A generic function $\mathbf{v}_{h} \in \boldsymbol{V}_{k, h}^{\text {face }}(P)$ is virtual so we can not use it directly. To proceed with the VEM discretization of Problem (11), we show that it is possible to compute some useful quantities.

- We can explicitly compute the polynomial $\left(\mathbf{v}_{h} \cdot \mathbf{n}_{f}\right)$ for each face $f$ on $\partial P$.

Since $\left(\mathbf{v}_{h} \cdot \mathbf{n}_{f}\right) \in \mathbb{P}_{k}(f)$, we can write it in terms of the monomial basis $\mathcal{M}_{k}(f)$. We exploit the normal face moments to find all coefficients $c_{i}$ of such polynomial, i.e. we write such polynomial as

$$
\left(\mathbf{v}_{h} \cdot \mathbf{n}_{f}\right)=\sum_{|\gamma|=0}^{k} c_{i} m_{\gamma}^{f}
$$


and we test it against a each element of $\mathcal{M}_{k}(f)$

$$
\sum_{|\gamma|=0}^{k} c_{i} \int_{f} m_{\gamma}^{f} m_{\boldsymbol{\beta}}^{f} \mathrm{~d} f=\int_{f}\left(\mathbf{v}_{h} \cdot \mathbf{n}_{f}\right) m_{\boldsymbol{\beta}}^{f} \mathrm{~d} f, \quad \forall m_{\boldsymbol{\beta}}^{f} \in \mathcal{M}_{k}(f) .
$$

Starting from these relations, we find exactly $\left(\mathbf{v}_{h} \cdot \mathbf{n}_{f}\right)$ on each face of $\partial P$;

- We can explicitly compute the divergence of a virtual function $\mathbf{v}_{h}$.

Since $\operatorname{div}\left(\mathbf{v}_{h}\right) \in \mathbb{P}_{k-1}(P)$, we can write it in terms of the monomial basis $\mathcal{M}_{k-1}(P)$, i.e.

$$
\operatorname{div}\left(\mathbf{v}_{h}\right)=\sum_{|\gamma|=0}^{k-1} c_{i} m_{\gamma}
$$

then, to get the coefficients $c_{i}$ of such polynomial, we test it against each term of the scaledmonomial basis of degree $k-1$

$$
\sum_{|\gamma|=0}^{k-1} c_{i} \int_{P} m_{\gamma} m_{\boldsymbol{\alpha}} \mathrm{d} P=\int_{P} \operatorname{div}\left(\mathbf{v}_{h}\right) m_{\boldsymbol{\alpha}} \mathrm{d} P, \quad \forall m_{\boldsymbol{\alpha}} \in \mathcal{M}_{k-1}(P) .
$$

Even if $\mathbf{v}_{h}$ is virtual, it is possible to compute exactly the right hand sides starting from the internal face moments and internal gradient moments. Indeed, if we integrate by parts we have

$$
\int_{P} \operatorname{div}\left(\mathbf{v}_{h}\right) m_{\boldsymbol{\alpha}} \mathrm{d} P=-\int_{P} \mathbf{v}_{h} \cdot \nabla m_{\boldsymbol{\alpha}}+\sum_{f \in \partial P} \int_{f}\left(\mathbf{v}_{h} \cdot \mathbf{n}_{f}\right) m_{\boldsymbol{\alpha}} \mathrm{d} f,
$$

the first integral is an internal gradient moment and we can find the polynomial $\left(\mathbf{v}_{h} \cdot \mathbf{n}_{f}\right)$ from the internal face degrees of freedom, see the previous item.

- We can compute an $L^{2}$-projection operator.

We define the $L^{2}$-projection operator $\boldsymbol{\Pi}_{k}^{0}: \boldsymbol{V}_{k, h}^{\text {face }}(P) \rightarrow\left[\mathbb{P}_{k}(P)\right]^{3}$ via

$$
\int_{P} \boldsymbol{\Pi}_{k}^{0} \mathbf{v}_{h} \cdot \boldsymbol{p}_{k} \mathrm{~d} P=\int_{P} \mathbf{v}_{h} \cdot \boldsymbol{p}_{k} \mathrm{~d} P, \quad \forall \boldsymbol{p}_{k} \in \mathbb{P}_{k}(P) .
$$

To compute such projection operator, one considers the vectorial monomial base $\left[\mathcal{M}_{k}(P)\right]^{3}$ for the projection $\boldsymbol{\Pi}_{k}^{0} \mathbf{v}_{h}$, i.e.

$$
\boldsymbol{\Pi}_{k}^{0} \mathbf{v}_{h}=\sum_{i=1}^{n_{k}} c_{i} \mathbf{m}_{i}
$$

where $n_{k}=\operatorname{dim}\left(\left[\mathcal{M}_{k}(P)\right]^{3}\right)$, and the relations

$$
\sum_{i=1}^{n_{k}} \int_{P} \mathbf{m}_{i} \cdot \mathbf{m}_{j} \mathrm{~d} P=\int_{P} \mathbf{v}_{h} \cdot \mathbf{m}_{j} \mathrm{~d} P, \quad \forall \mathbf{m}_{j} \in\left[\mathcal{M}_{k}(P)\right]^{3}
$$

to find the unknown coefficients $c_{i}$. The right hand side of such conditions involves virtual function so we have to understand if it is computable. Exploiting Propositions 2.1, 2.2, 2.3 and 2.5, we have

$$
\begin{aligned}
\int_{P} \mathbf{v}_{h} \cdot \mathbf{m}_{j} \mathrm{~d} P & =c_{1} \int_{P} \mathbf{v}_{h} \cdot \nabla m_{\boldsymbol{\beta}} \mathrm{d} P+c_{2} \int_{P} \mathbf{v}_{h} \cdot\left(\mathbf{m}_{\mathbf{I}} \wedge \overline{\mathbf{m}}\right) \mathrm{d} P \\
& +c_{3} \int_{P} \mathbf{v}_{h} \cdot\left(\mathbf{m}_{\mathbf{I}} \wedge \widetilde{\mathbf{m}}\right) \mathrm{d} P
\end{aligned}
$$

where $c_{1}, c_{2}$ and $c_{3}$ are suitable constant, $m_{\boldsymbol{\beta}}, \widetilde{\mathbf{m}}$ and $\overline{\mathbf{m}}$ are proper scaled monomials to decompose the vectorial monomial $\mathbf{m}_{j}$. The last two integrals in Equation (17) are internal cross 
moments degrees of freedom. The first integral is a gradient moment only if $|\boldsymbol{\beta}| \leq k-1$, otherwise we integrate by parts and get

$$
\int_{P} \mathbf{v}_{h} \cdot \nabla m_{\boldsymbol{\beta}} \mathrm{d} P=-\int_{P} \operatorname{div}\left(\mathbf{v}_{h}\right) m_{\boldsymbol{\beta}}+\sum_{f \in \partial P} \int_{f}\left(\mathbf{v}_{h} \cdot \mathbf{n}_{f}\right) m_{\boldsymbol{\beta}} \mathrm{d} f,
$$

which is still computable since we know both $\operatorname{div}\left(\mathbf{v}_{h}\right)$ and $\left(\mathbf{v}_{h} \cdot \mathbf{n}_{f}\right)$ on each face of $P$.

Then, the discrete velocity global space is defined by gluing such local spaces, i.e.

$$
\boldsymbol{V}_{k, h}\left(\Omega_{h}\right):=\left\{\mathbf{v}_{h} \in H^{1}(\operatorname{div}, \Omega):\left.\mathbf{v}_{h}\right|_{P} \in \boldsymbol{V}_{k, h}^{\mathrm{face}}(P) \forall P \in \Omega_{h}, \mathbf{v}_{h} \cdot \mathbf{n}=u_{N}, \text { on } \partial \Omega\right\} .
$$

\subsection{Definition of $Q_{k-1, h}\left(\Omega_{h}\right)$}

To discretize the pressure, we consider a discontinuous polynomial space defined on each polyhedron $P$ of the discretization $\Omega_{h}$. Given a polyhedron $P$, we introduce the local space

$$
Q_{k-1, h}(P):=\left\{q_{h} \in L^{2}(P): q_{h} \in \mathbb{P}_{k-1}(P)\right\} .
$$

The degrees of freedom of such space are

- internal moments

$$
\frac{1}{|P|} \int_{P} q_{h} m_{\boldsymbol{\alpha}} \mathrm{d} P \quad \forall m_{\boldsymbol{\alpha}} \in \mathcal{M}_{k-1}(P) .
$$

Since the a function $q_{h} \in Q_{k-1, h}(P)$ is a polynomial of degree $k-1$, it can be computed exactly on the polyhedron $P$ via the internal moments so we do not need to define any projection operator.

Then, as we have done for the global velocity discrete space, the global pressure space is defined by gluing local spaces, i.e.

$$
Q_{k-1, h}\left(\Omega_{h}\right):=\left\{q_{h} \in L^{2}(\Omega):\left.q_{h}\right|_{P} \in Q_{k-1, h}(P), \quad \forall P \in \Omega_{h} \text { and } \int_{\Omega} q_{h} d x=0\right\} .
$$

\subsection{The discrete local forms}

To proceed with the discretization of Problem (11), we construct suitable discrete forms [15, 13, 20]. As in standard VEM approach such discrete forms are defined element-wise and they depend on the degrees of freedom and projection operators.

Recalling Equation (12), we define

$$
\begin{aligned}
\boldsymbol{a}_{h, P}\left(\mathbf{v}_{h}, \boldsymbol{w}_{h}\right) & :=\nu \int_{P} \boldsymbol{\Pi}_{k}^{0} \mathbf{v}_{h} \cdot \boldsymbol{\Pi}_{k}^{0} \boldsymbol{w}_{h} \mathrm{~d} P+s_{P}\left(\mathbf{v}_{h}-\Pi_{k}^{0} \mathbf{v}_{h}, \boldsymbol{w}_{h}-\boldsymbol{\Pi}_{k}^{0} \boldsymbol{w}_{h}\right), \\
\boldsymbol{b}_{h, P}\left(\mathbf{v}_{h}, q_{h}\right) & :=\int_{P} \operatorname{div}\left(\mathbf{v}_{h}\right) q_{h} \mathrm{~d} P \\
\mathbf{f}_{h, P}\left(q_{h}\right) & :=\int_{P} f q_{h} \mathrm{~d} P
\end{aligned}
$$

where $\mathbf{v}_{h}, \boldsymbol{w}_{h} \in \boldsymbol{V}_{k, h}^{\text {face }}(P), q_{h} \in Q_{k-1, h}(P)$ and $s_{P}$ can be any symmetric and positive definite bilinear form which scales as the $\boldsymbol{a}_{P}(\cdot, \cdot)$. Such operator has to verify that there exist two constant $\alpha_{*}, \alpha^{*}>0$ such that

$$
\alpha_{*} a_{P}\left(\mathbf{v}_{h}, \mathbf{v}_{h}\right) \leq s_{P}\left(\mathbf{v}_{h}, \mathbf{v}_{h}\right) \leq \alpha^{*} a_{P}\left(\mathbf{v}_{h}, \mathbf{v}_{h}\right),
$$

the coefficients $\alpha_{*}, \alpha^{*}$ depend on $\nu$ but not on the mesh-size. In this paper we choose the Euclidean scalar product associated with the degrees of freedom of $\boldsymbol{V}_{k, h}^{\text {face }}(P)$ multiplied by the volume of $P$ and the value of $\nu$ at its barycenter [13, 20, i.e.

$$
s_{P}\left(\mathbf{v}_{h}, \boldsymbol{w}_{h}\right):=\nu\left(\boldsymbol{x}_{P}\right)|P| \sum_{i=1}^{\# \operatorname{dof}_{P}} \operatorname{dof} f_{i}\left(\mathbf{v}_{h}\right) \operatorname{dof}\left(\boldsymbol{w}_{h}\right),
$$

where $\# d o f_{P}$ are the number of degrees of freedom associated with a function in $\boldsymbol{V}_{k, h}^{\text {face }}(P)$ and $d o f_{i}$ : $\boldsymbol{V}_{k, h}^{\mathrm{face}}(P) \rightarrow \mathbb{R}$ is a linear functional which associate to a function in $\boldsymbol{V}_{k, h}^{\mathrm{face}}(P)$ the value of its $i-$ th degree of freedom. 
Remark 4.2. The operators $\boldsymbol{b}_{h, P}$ and $\mathbf{f}_{h, P}$ involves polynomials ( $\operatorname{div}\left(\mathbf{v}_{h}\right)$ and $q_{h}$ ) and the datum $f$. Consequently, the discrete approximations of such terms are related only on the computation of integrals, i.e. on the quadrature formulas used.

Once we have defined the global forms

$$
\begin{aligned}
\boldsymbol{a}_{h}\left(\mathbf{v}_{h}, \boldsymbol{w}_{h}\right) & :=\sum_{P \in \Omega_{h}} \boldsymbol{a}_{h, P}\left(\mathbf{v}_{h}, \boldsymbol{w}_{h}\right) \\
\boldsymbol{b}_{h}\left(\mathbf{v}_{h}, q_{h}\right) & :=\sum_{P \in \Omega_{h}} \boldsymbol{b}_{h, P}\left(\mathbf{v}_{h}, q_{h}\right) \\
\mathbf{f}_{h}\left(q_{h}\right) & :=\sum_{P \in \Omega_{h}} \mathbf{f}_{h, P}\left(q_{h}\right),
\end{aligned}
$$

the discrete variational problem reads

$$
\left\{\begin{array}{rlrl}
\text { find }\left(\mathbf{u}_{h}, p_{h}\right) \in \boldsymbol{V}_{k, h}\left(\Omega_{h}\right) \times Q_{k-1, h}\left(\Omega_{h}\right) & & \\
\boldsymbol{a}_{h}\left(\mathbf{u}_{h}, \mathbf{v}_{h}\right)-\boldsymbol{b}_{h}\left(\mathbf{v}_{h}, p_{h}\right) & =0 & & \forall \mathbf{v}_{h} \in \boldsymbol{V}_{k, h}\left(\Omega_{h}\right) \\
\boldsymbol{b}_{h}\left(\mathbf{u}_{h}, q_{h}\right) & =\left(\mathbf{f}_{h}, q_{h}\right) & & \forall q_{h} \in Q_{k-1, h}\left(\Omega_{h}\right) .
\end{array}\right.
$$

Remark 4.3. Consider a polyhedral mesh $\Omega_{h}$ where all the elements are uniformly star shaped with respect to a ball and all the edge/face diameters are comparable with respect to the polyhedron diameters. Under these assumptions Problem (20) has a unique solution $\left(\mathbf{v}_{h}, q_{h}\right) \in \boldsymbol{V}_{k, h}\left(\Omega_{h}\right) \times Q_{k-1, h}\left(\Omega_{h}\right)$ satisfying the following error estimates:

$$
\begin{aligned}
\left\|\mathbf{u}-\mathbf{u}_{h}\right\|_{0} & \leq C h^{k}\left(\|\mathbf{u}\|_{k+1}+\|q\|_{k+1}\right), \\
\left\|q-q_{h}\right\|_{0} & \leq C h^{k+1}\left(\|\mathbf{u}\|_{k+1}+\|q\|_{k+1}\right), \\
\left\|\operatorname{div}\left(\mathbf{u}-\mathbf{u}_{h}\right)\right\|_{0} & \leq C h^{k+1}\left(|f|_{k+1}+\|q\|_{k+1}\right),
\end{aligned}
$$

where $C$ is a constant depending on $\nu$ but independent from the mesh size $h$. The proof is beyond the scope of this paper and it could be obtained by combining the results in [33, 28].

\subsection{Numerical results}

We conclude this section with a numerical example to validate the mixed virtual element approach in solving Problem (11). In the following test we use four different discretizations of the cube $[0,1]^{3}$ :

- Cube, a mesh composed by structured cubes,

- Octa, a mesh composed by polyhedron with seven or eight faces,

- CVT, a Voronoi tessellation optimized via a standard Llyod algorithm [35],

- Random a Voronoi tessellation of a set of points randomly put inside $\Omega$.

In such discretizations the mesh elements become more irregular. Indeed, firstly we take into account standard cubes and regularly shaped polyhedrons, Cube and Octa meshes. Then, we move to CVT and Random meshes which are characterized by small edges, stretched and small faces, see Figure 1 .

All the meshes taken into account were generated via the c++ library voro++ [45] and putting the control points of the Voronoi cells in a proper way [14, 35]. In order to study the error convergence rate, we generate a sequence of four progressive refinements composed by approximately 27, 125, 1000 and 8000 polyhedrons and we associate with them a mesh-size

$$
h:=\frac{1}{N_{P}} \sum_{i=1}^{N_{P}} h_{P}
$$

where $N_{P}$ is the number of polyhedrons in the mesh. 


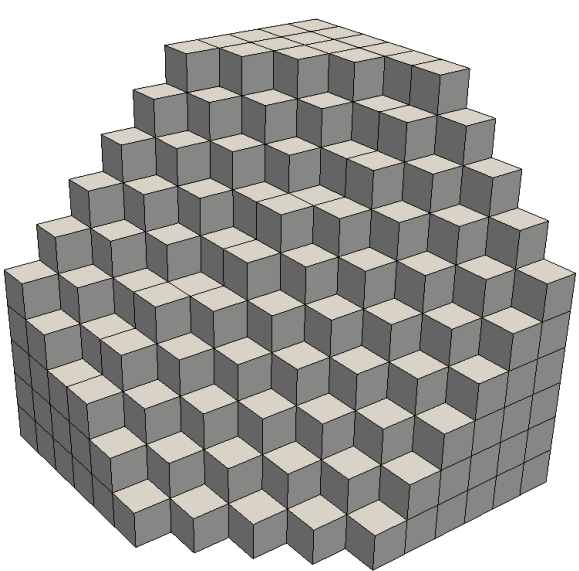

Cube

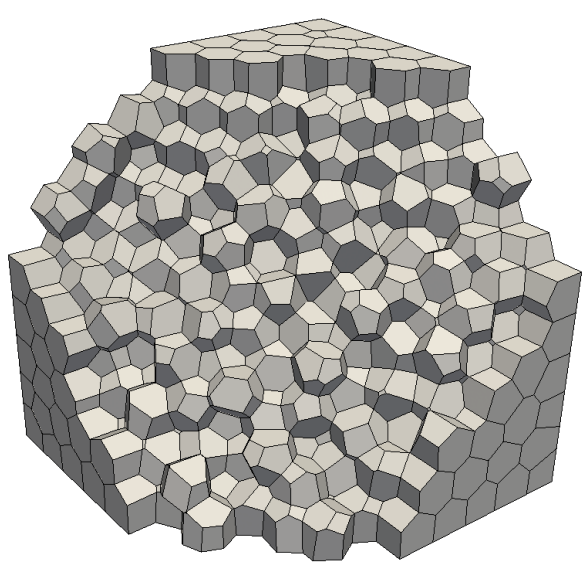

CVT

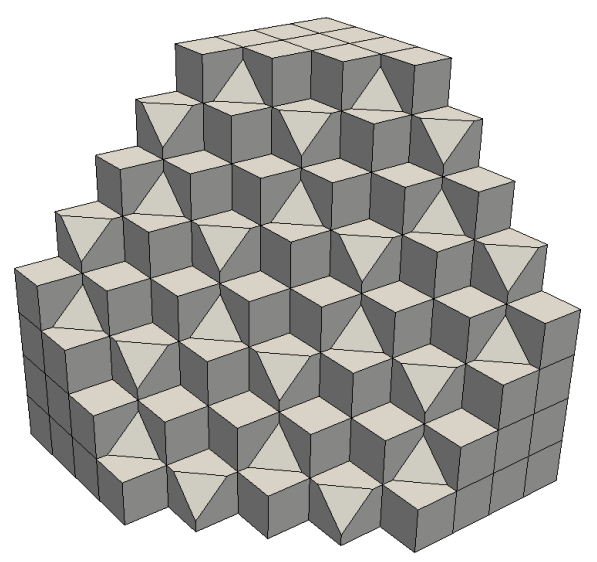

Octa

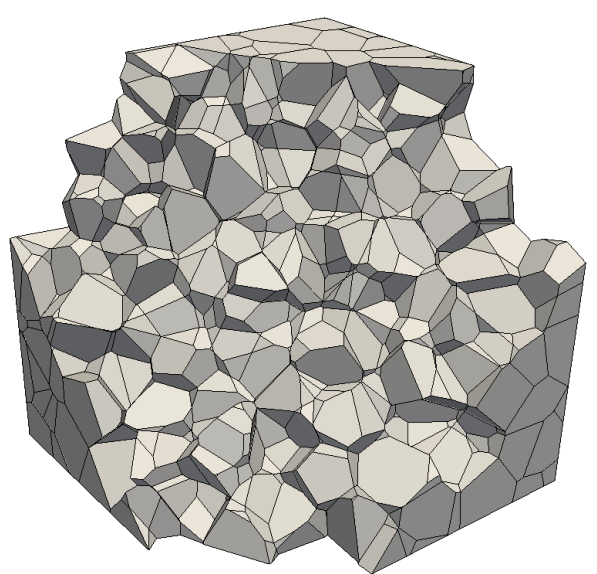

Random

Figure 1: A sample of the mesh taken into account.

We compute the error of both velocity and pressure. More specifically we consider the following $L^{2}$ error indicators

$$
e_{\mathbf{v}}:=\frac{\sqrt{\sum_{i=1}^{N_{P}}\left\|\mathbf{v}-\boldsymbol{\Pi}_{k}^{0} \mathbf{v}_{h}\right\|_{0, P}^{2}}}{\|\mathbf{v}\|_{0, \Omega}}, \quad e_{q}:=\frac{\sqrt{\sum_{i=1}^{N_{P}}\left\|q-q_{h}\right\|_{0, P}^{2}}}{\|q\|_{0, \Omega}},
$$

where $\|\cdot\|_{0, \mathcal{D}}$ denotes the standard $L^{2}$ norm over a domain $\mathcal{D}$.

We remark that the function $\mathbf{v}_{h}$ is virtual so we exploit its projection to compute the error $e_{\mathbf{v}}$. On the other hand the discrete function associated with the pressure is a polynomial of degree $k-1$ on each element and we do not use any projection operator to compute such error. The expected convergence rates of $e_{\mathbf{v}}$ and $e_{q}$ are $O\left(h^{k}\right)$ and $O\left(h^{k+1}\right)$, respectively, see Remark 4.3 .

We consider the Problem (11) with $\nu(\boldsymbol{x})=1$ and we set both right hand side and the boundary conditions in such a way that the exact solution is the couple

$$
\mathbf{v}(x, y, z):=\left(\begin{array}{c}
-5 x^{4}-y^{2} z^{3} \\
-24 y^{3}-2 x y z^{3} \\
-27 z^{2}-3 x y^{2} z^{2}
\end{array}\right)
$$

and

$$
q(x, y, z):=x^{5}+6 y^{4}+9 z^{3}+x y^{2} z^{3} .
$$

In Figure 2 we show the convergence lines for all meshes and for $k=1,2,3$ and 4 . For all the set of meshes and for each approximation degree $k$, the method behaves as expected and we recover the convergence rate predicted by the theory for both velocity and pressure. 
Moreover, if we fix the degree $k$ and we vary the type of meshes, these convergence lines are close to each other. This fact is a further numerical prove of the robustness of VEM with respect to distorted elements.

The method fails only in the last step of the Random meshes for the error $e_{\mathbf{v}}$. This fact could be due to the ill-conditioning of the linear system at hand. Indeed, such mesh is characterized by really small features (the smallest face has area $3.9 \times 10^{-14}$ and the smallest edge is long $5.9 \times 10^{-8}$ ) which may affect the condition number of the stiffness matrix and consequently the computation of the error, when we have such an high approximation degree.
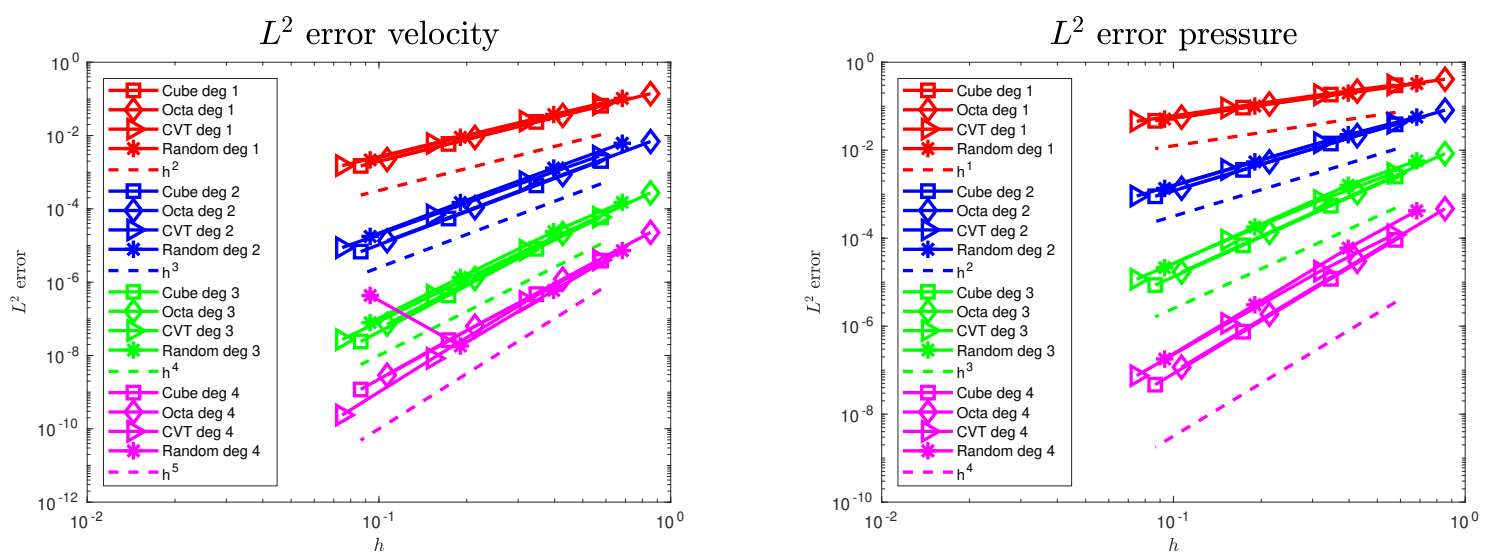

Figure 2: Convergence lines for each set of meshes taken into account and degrees $k=2,3$ and 4 .

\section{Parallel preconditioners}

Our strategy for building an efficient parallel solver is based on the parallel library PETSc from Argonne National Laboratory [10, 11, 12]. Such library is built on the MPI standard and it offers advanced data structures and routines for the parallel solution of partial differential equations, from basic vector and matrix operations to more complex linear and nonlinear equation solvers. In our $\mathrm{c}++$ code, vectors and matrices are built and subassembled in parallel on each processor.

Let us denote by $\mathcal{A}$ the linear system matrix arising from the discretization of the model problem (11), which has the typical saddle point structure

$$
\mathcal{A}=\left[\begin{array}{cc}
A & B^{T} \\
B & -C
\end{array}\right]
$$

To solve such linear system, we use the parallel GMRES method provided by the PETSc library, preconditioned by two types of block-diagonal preconditioners (see e.g. [25, 40, 8]) of the form

$$
\mathcal{B}_{D}=\left[\begin{array}{cc}
\mathrm{B}_{1} & 0 \\
0 & \mathrm{~B}_{2}
\end{array}\right]
$$

- Block-Schur where

$$
\begin{aligned}
& B_{1}^{-1}=\text { diagonal preconditioner for } A \text {, i.e. } B_{1}=\operatorname{diag}(A) \\
& B_{2}^{-1}=\text { exact solution of the approximate Schur complement } S
\end{aligned}
$$

with $S=-C-B \operatorname{diag}(A)^{-1} B^{T}$. For the inversion of $S$ at each preconditioning step we use the parallel multifrontal direct solver Mumps [1, 2].

- Block-Reg where

$$
\begin{aligned}
& B_{1}^{-1}=\text { Algebraic Multigrid preconditioner for } A+B^{T} W^{-1} B, \\
& B_{2}^{-1}=W^{-1}
\end{aligned}
$$

with $W=\gamma I$, for a suitable parameter $\gamma>0$. As Algebraic Multigrid preconditioner we use the GAMG solver of PETSc. 
In the following tests we compare the previous two block-diagonal preconditioners and the parallel direct solver Mumps considering the model problem in Subsection 4.4 .

\subsection{Numerical results}

In the numerical tests we use the Linux cluster INDACO (www.indaco.unimi.it) of the University of Milan, constituted by 16 nodes, each carrying 2 processors INTEL XEON E5-2683 V4 $2.1 \mathrm{GHz}$, with 16 cores each.

We consider three types of polyhedral meshes, Cube, Octa and CVT, introduced in Subsection 4.4. We solve our model problem (11) using the proposed VEM discretizations. For the Block-Reg preconditioner, we heuristically found that the best performances are obtained taking $\gamma=h^{2}$, where $h$ is the mesh size parameter defined in Equation 21.

\begin{tabular}{|c|c|c|c|c|c|c|c|c|c|c|c|}
\hline \multicolumn{12}{|c|}{ Cube mesh with 32768 elements, $\mathrm{k}=1$, dofs $=435201$} \\
\hline \multirow[t]{2}{*}{$p$} & \multirow{2}{*}{$S_{p}^{\text {id }}$} & \multirow[t]{2}{*}{$T_{\text {ass }}$} & \multirow[t]{2}{*}{$S_{p}$} & \multicolumn{2}{|c|}{ Mumps } & \multicolumn{3}{|c|}{ Block-Schur } & \multicolumn{3}{|c|}{ Block-Reg } \\
\hline & & & & $T_{\text {sol }}$ & $S_{p}$ & it & $T_{\text {sol }}$ & $S_{p}$ & it & $T_{\text {sol }}$ & $S_{p}$ \\
\hline 1 & - & 68 & - & 823 & - & 66 & 50 & - & 84 & 53 & 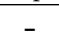 \\
\hline 4 & 4 & 19 & 3.6 & 228 & 3.6 & 66 & 17 & 2.9 & 114 & 32 & 1.7 \\
\hline 8 & 8 & 10 & 6.8 & 138 & 6.0 & 66 & 12 & 4.2 & 116 & 29 & .8 \\
\hline 16 & 16 & 5 & 13.6 & 74 & 11.1 & 66 & 10 & 5.0 & 120 & 26 & 2.0 \\
\hline 32 & 32 & 3 & 22.7 & 47 & 17.5 & 66 & 9 & 5.5 & 137 & 26 & 2.0 \\
\hline \multicolumn{12}{|c|}{ Cube mesh with 13824 elements, $\mathrm{k}=2$, dofs $=508033$} \\
\hline \multirow[t]{2}{*}{$p$} & \multirow[t]{2}{*}{$S_{p}^{\text {id }}$} & \multirow[t]{2}{*}{$T_{a s s}$} & \multirow[t]{2}{*}{$S_{p}$} & \multicolumn{2}{|c|}{ Mumps } & \multicolumn{3}{|c|}{ Block-Schur } & \multicolumn{3}{|c|}{ Block-Reg } \\
\hline & & & & $T_{\text {sol }}$ & $S_{p}$ & it & $T_{\text {sol }}$ & $S_{p}$ & it & $T_{\text {sol }}$ & $S_{p}$ \\
\hline 1 & - & 271 & - & 659 & - & 72 & 347 & - & 98 & 138 & - \\
\hline 4 & 4 & 97 & 2.8 & 436 & 1.5 & 72 & 118 & 2.9 & 162 & 73 & 1.9 \\
\hline 8 & 8 & 53 & 5.1 & 270 & 2.4 & 72 & 77 & 4.5 & 168 & 48 & 2.9 \\
\hline 16 & 16 & 27 & 10.0 & 157 & 4.2 & 72 & 55 & 6.3 & 172 & 67 & 2.1 \\
\hline 32 & 32 & 13 & 20.8 & 95 & 6.9 & 72 & 40 & 8.7 & 182 & 58 & 2.4 \\
\hline \multicolumn{12}{|c|}{ Cube mesh with 8000 elements, $\mathrm{k}=3$, dofs $=612001$} \\
\hline \multirow[t]{2}{*}{$p$} & \multirow[t]{2}{*}{$S_{p}^{\text {id }}$} & \multirow[t]{2}{*}{$T_{a s s}$} & \multirow[t]{2}{*}{$S_{p}$} & \multicolumn{2}{|c|}{ Mumps } & \multicolumn{3}{|c|}{ Block-Schur } & \multicolumn{3}{|c|}{ Block-Reg } \\
\hline & & & & $T_{\text {sol }}$ & $S_{p}$ & it & $T_{\text {sol }}$ & $S_{p}$ & it & $T_{\text {sol }}$ & $S_{p}$ \\
\hline 4 & - & 623 & - & 461 & - & 147 & 570 & - & 720 & 450 & - \\
\hline 8 & 2 & 33 & 1.9 & 277 & 1.7 & 147 & 430 & 1.3 & 784 & 285 & 1.6 \\
\hline 16 & 4 & 194 & 3.2 & 163 & 2.8 & 147 & 268 & 2.1 & 774 & 203 & 2.2 \\
\hline 32 & 8 & 97 & 6.4 & 99 & 4.7 & 147 & 192 & 3.0 & 918 & 197 & 2.3 \\
\hline
\end{tabular}

Table 1: Strong scaling test, Cube meshes. $p:=$ number of procs; $S_{p}^{\text {id }}:=$ ideal speedup; $T_{a s s}:=$ assembling time in seconds; $T_{\text {sol }}:=$ solution time in seconds; it:=GMRES iterations; $S_{p}:=$ parallel speedup computed with respect to the 1 procs run for $\mathrm{k}=1,2$ and to the 4 procs run for $\mathrm{k}=3$.

\subsubsection{Test 1: strong scaling}

In this paragraph, we study the parallel performance of the three solvers (Mumps, Block-Schur and Block-Reg), by increasing the number of processors, while keeping fixed the global number of degrees of freedom (dofs). Hence, this is a strong scaling test. For all the three meshes, we consider a VEM discretizations of order $k=1,2,3$.

The results of the strong scaling test are displayed in Tables 1, 3 and 2, Note that, in case of order $k=3$, the runs with 1 processor (procs) went Out of Memory. Thus, we report the results starting from the 4 procs run. Denoting by $p$ the number of procs, we recall that the parallel speedup $S_{p}$ is defined as

$$
S_{p}:=\frac{\text { CPU time with } 1 \text { procs }}{\text { CPU time with } p \text { procs }}
$$



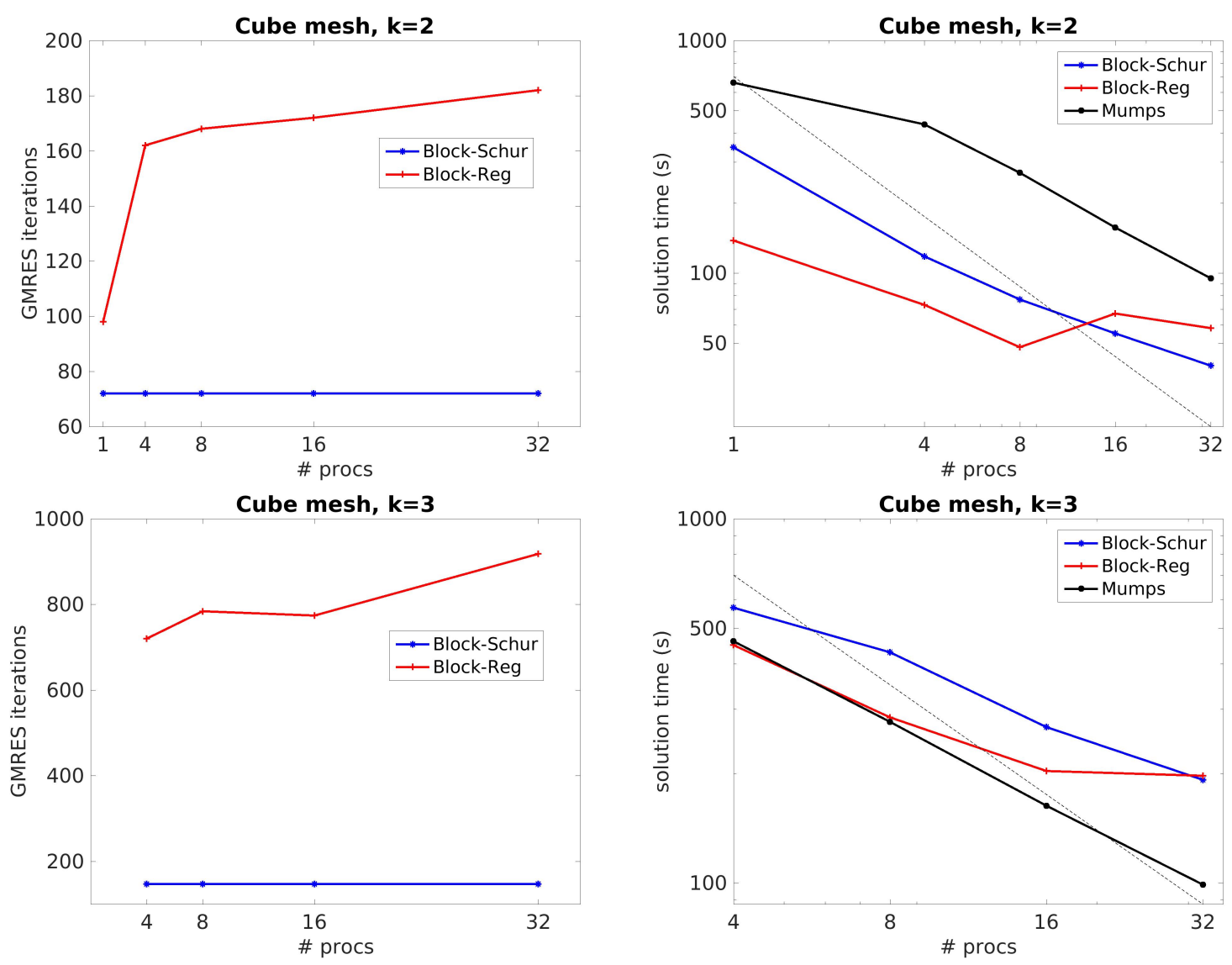

Figure 3: Strong scaling test, Cube meshes. GMRES iterations (left) and solution time (right) as a function of the number of procs for $k=2$ (first row) and $k=3$ (second row). In the solution time plots, the black dashed line indicates the steepness of the ideal time reduction.

We first observe that, irrespective of the kind of mesh and of the order $k$ of the VEM discretization, the CPU times needed to assemble the stiffness matrix and the right hand side $\left(T_{\text {ass }}\right)$ are scalable, with good speedup values quite close to the ideal ones.

In case of Cube meshes (Table 1 and Fig. 3), for $k=1,2$, the Block-Schur preconditioner is completely scalable in terms of GMRES iterations, which do not depend on $p$, whereas the Block-Reg preconditioner shows a slight increase of GMRES iterations. The solution times $\left(T_{\text {sol }}\right)$ decrease with $p$ for all the three solvers, but the speedup values are far from the ideal ones. The highest speedup values are achieved by Mumps for $k=1$ and Block-Schur for $k=2$. In terms of CPU times, for both $k=1,2$, the most effective solver is Block-Schur, being between 2 and 16 times as fast as Mumps. For $k=3$ instead, the iterative solvers suffer in terms of GMRES iterations, due to the severe ill-conditioning of the stiffness matrix. Mumps is the most effective solver, being twice as fast as the iterative methods on 32 procs.

In case of Octa meshes (Table 2 and Fig. 4), the GMRES iterations of the iterative solvers are higher (much higher for $k=2,3$ ) than in case of Cube meshes, even though the global problem is comparable or even smaller in terms of dofs, indicating that the condition number of the stiffness matrix associated to the Octa mesh is worse than that of the Cube meshes. For $k=1,2$, the BlockSchur preconditioner is completely scalable in terms of iterations, while Block-Reg presents a slight increase. In terms of CPU times, for $k=1$, the most effective solver is Block-Schur, which is between 9 and 30 times as fast as Mumps. For $k=2$ instead, differently from the Cube test, Mumps is very effective on Octa meshes and results to be the fastest solver, presenting also a good scalability. Note that, with 32 procs and $k=3$, Mumps is 25 and 7 times as fast as the Block-Schur and Block-Reg preconditioners, respectively.

In case of CVT meshes (Table 3 and Fig. 5), for $k=1,2$ the performance of the Block-Schur and Block-Reg solvers is comparable with the case of the Octa meshes, in terms of GMRES iterations. 
In terms of CPU time, the speedup values are far from the ideal ones, but the iterative solvers are much more effective than Mumps, which fails for $k=2$ due to Out of Memory for $p=1,4$ and is very slow for $p=8,16,32$. Indeed, for $k=1,2$, the Block-Schur solver results to be between 9 and 300 times as fast as Mumps. For $k=3$ instead, the behavior of the iterative solvers degenerate, due to the severe ill-conditioning of the stiffness matrix. Consequently, Mumps becomes competitive or even faster than the iterative methods.

\begin{tabular}{|c|c|c|c|c|c|c|c|c|c|c|c|}
\hline \multicolumn{12}{|c|}{ Octa mesh with 30375 elements, $\mathrm{k}=1$, dofs $=453601$} \\
\hline \multirow{2}{*}{$p$} & \multirow{2}{*}{$S_{p}^{\text {id }}$} & \multirow[t]{2}{*}{$T_{\text {ass }}$} & \multirow{2}{*}{$S_{p}$} & \multicolumn{2}{|c|}{ Mumps } & \multicolumn{3}{|c|}{ Block-Schur } & \multicolumn{3}{|c|}{ Block-Reg } \\
\hline & & & & $T_{\text {sol }}$ & $S_{p}$ & it & $T_{\text {sol }}$ & $S_{p}$ & it & $T_{\text {sol }}$ & $S_{p}$ \\
\hline 1 & - & 79 & - & 1901 & - & 79 & 60 & - & 93 & 61 & - \\
\hline 4 & 4 & 26 & 3.0 & 551 & 3.4 & 79 & 21 & 2.9 & 239 & 65 & 0.9 \\
\hline 8 & 8 & 14 & 5.6 & 336 & 5.7 & 79 & 17 & 3.5 & 240 & 50 & 1.2 \\
\hline 16 & 16 & 7 & 11.3 & 205 & 9.3 & 79 & 12 & 5.0 & 246 & 44 & 1.4 \\
\hline 32 & 32 & 3 & 26.3 & 116 & 16.4 & 79 & 13 & 4.6 & 255 & 33 & 1.8 \\
\hline \multicolumn{12}{|c|}{ Octa mesh with 9000 elements, $\mathrm{k}=2$, dofs $=361201$} \\
\hline \multirow[t]{2}{*}{$p$} & \multirow[t]{2}{*}{$S_{p}^{\text {id }}$} & \multirow[t]{2}{*}{$T_{\text {ass }}$} & \multirow[t]{2}{*}{$S_{p}$} & \multicolumn{2}{|c|}{ Mumps } & \multicolumn{3}{|c|}{ Block-Schur } & \multicolumn{3}{|c|}{ Block-Reg } \\
\hline & & & & $T_{\text {sol }}$ & $S_{p}$ & it & $T_{\text {sol }}$ & $S_{p}$ & it & $T_{\text {sol }}$ & $S_{p}$ \\
\hline 1 & - & 342 & - & 326 & 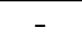 & 559 & 276 & - & 113 & 109 & - \\
\hline 4 & 4 & 97 & 3.5 & 107 & 3.0 & 559 & 113 & 2.4 & 233 & 83 & 1.3 \\
\hline 8 & 8 & 60 & 5.7 & 66 & 4.9 & 559 & 102 & 2.7 & 277 & 58 & 1.9 \\
\hline 16 & 16 & 32 & 10.7 & 38 & 8.6 & 559 & 83 & 3.3 & 274 & 61 & 1.8 \\
\hline 32 & 32 & 16 & 21.4 & 24 & 13.6 & 559 & 84 & 3.3 & 285 & 46 & 2.4 \\
\hline \multicolumn{12}{|c|}{ Octa mesh with 4608 elements, $\mathrm{k}=3$, dofs $=378881$} \\
\hline \multirow[t]{2}{*}{$p$} & \multirow[t]{2}{*}{$S_{p}^{\text {id }}$} & \multirow[t]{2}{*}{$T_{\text {ass }}$} & \multirow[t]{2}{*}{$S_{p}$} & \multicolumn{2}{|c|}{ Mumps } & \multicolumn{3}{|c|}{ Block-Schur } & \multicolumn{3}{|c|}{ Block-Reg } \\
\hline & & & & $T_{\text {sol }}$ & $S_{p}$ & it & $T_{\text {sol }}$ & $S_{p}$ & it & $T_{\text {sol }}$ & $S_{p}$ \\
\hline 4 & - & 683 & - & 108 & - & 2282 & 918 & - & 1177 & 421 & - \\
\hline 8 & 2 & 354 & 1.9 & 75 & 1.4 & 2503 & 746 & 1.2 & 1436 & 316 & 1.3 \\
\hline 16 & 4 & 192 & 3.6 & 44 & 2.4 & 2188 & 609 & 1.5 & 1561 & 257 & 1.6 \\
\hline 32 & 8 & 98 & 7.0 & 26 & 4.1 & 2192 & 660 & 1.4 & 1618 & 180 & 2.3 \\
\hline
\end{tabular}

Table 2: Strong scaling test, Octa meshes. Same format as in Table 1.

\subsubsection{Test 2: optimality}

In this test, we investigate the behavior of the parallel solvers when refining the mesh size, thus increasing the number of dofs. The number of processors $p$ and the order of VEM discretization $k$ are kept fixed to 8 and 2, respectively. The results are reported in Tables 4, 6, 5 and in Fig. 6. In terms of GMRES iterations, the Block-Schur preconditioner exhibits an optimal behavior, since the number of iterations remains bounded from above when varying the number of dofs. The BlockReg preconditioner instead shows a quasi-optimal behavior, with a growth of GMRES iterations that appears to be logarithmic.

On the largest Cube mesh (804385 dofs), the Block-Schur and Block-Reg solvers are about 3 and 7 times as fast as Mumps, respectively. On the largest Octa mesh (622081 dofs), Block-Reg results to be the most effective solver. On the largest CVT mesh (465721 dofs), the Block-Schur and Block-Reg solvers are about 19 and 17 times as fast as Mumps, respectively.

\section{Conclusion}

In this paper we constructed and numerically analyzed a general order VEM approximation scheme for three-dimensional scalar elliptic equations in mixed form. The convergence tests have demonstrated the effectiveness and the robustness of the proposed algorithms on different polyhedral grids. Moreover, we developed a parallel solver for the solution of the saddle point linear systems arising from the 

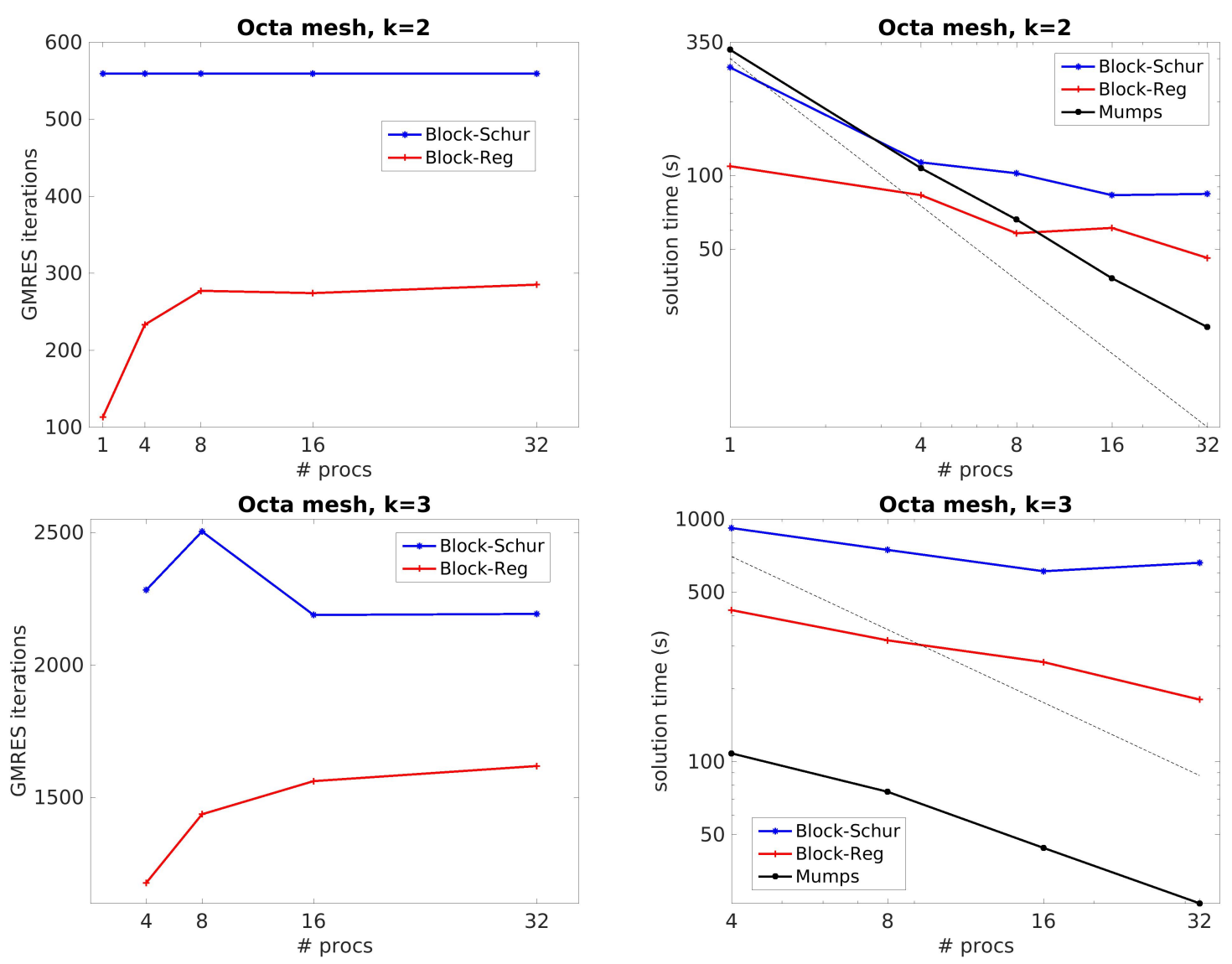

Figure 4: Strong scaling test, Octa meshes. Same format as in Fig. 3

discretization process, by exploiting both direct and iterative parallel solution methods, preconditioned by block-diagonal preconditioners. The numerical tests performed on a Linux cluster have shown that the proposed iterative methods are more effective than the Mumps direct solver for low order $(k=1,2)$ discretizations, while, using high order discretizations, they suffer due to the severe ill-conditioning of the linear system matrix and Mumps results to be the fastest solver. More research is needed in future to contruct preconditioners resulting to be robust with respect to high order of VEM discretizations. Further developments of the present investigation might be the construction and analysis of Balancing Domain Decomposition by Constraints (BDDC) preconditioners for VEM discretizations of elliptic equations in mixed form and the extension of the parallel solvers studied here to the solution of other saddle point linear systems arising from VEM discretizations of Stokes or Maxwell equations.

\section{Acknowledgments}

The authors would like to acknowledge INDAM-GNCS for the support. Moreover they would like to thank Lourenco Beirão da Veiga and Alessandro Russo for many helpful discussions and suggestions.

\section{References}

[1] P. R. Amestoy, I. S. Duff, J.-Y. L'Excellent, and J. Koster. A fully asynchronous multifrontal solver using distributed dynamic scheduling. SIAM J. Matr. Anal. Appl., 23(1):15-41, 2001.

[2] P. R. Amestoy, A. Guermouche, J.-Y. L'Excellent, and S. Pralet. Hybrid scheduling for the parallel solution of linear systems. Paral. Comput., 32(2):136-156, 2006.

[3] P. F. Antonietti, L. Beirão da Veiga, S. Scacchi, and M. Verani. A $C^{1}$ virtual element method for the Cahn-Hilliard equation with polygonal meshes. SIAM J. Numer. Anal., 54(1):34-57, 2016. 


\begin{tabular}{|c|c|c|c|c|c|c|c|c|c|c|c|}
\hline \multicolumn{12}{|c|}{ CVT mesh with 16000 elements, $\mathrm{k}=1$, dofs $=388043$} \\
\hline \multirow[t]{2}{*}{$p$} & \multirow{2}{*}{$S_{p}^{\text {id }}$} & \multirow{2}{*}{$T_{a s s}$} & \multirow{2}{*}{$S_{p}$} & \multicolumn{2}{|c|}{ Mumps } & \multicolumn{3}{|c|}{ Block-Schur } & \multicolumn{3}{|c|}{ Block-Reg } \\
\hline & & & & $T_{\text {sol }}$ & $S_{p}$ & it & $T_{\text {sol }}$ & $S_{p}$ & it & $T_{\text {sol }}$ & $S_{p}$ \\
\hline 1 & - & 164 & - & 10687 & 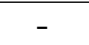 & 138 & 35 & - & 72 & 80 & - \\
\hline 4 & 4 & 52 & 3.1 & 2966 & 3.6 & 138 & 15 & 2.3 & 140 & 88 & 0.9 \\
\hline 8 & 8 & 27 & 6.1 & 1708 & 6.3 & 138 & 11 & 3.2 & 162 & 63 & 1.3 \\
\hline 16 & 16 & 14 & 11.7 & 1230 & 8.7 & 138 & 10 & 3.5 & 171 & 47 & 1.7 \\
\hline 32 & 32 & 7 & 23.4 & 614 & 17.4 & 138 & 12 & 2.9 & 179 & 32 & 2.5 \\
\hline
\end{tabular}

\begin{tabular}{|c|c|c|c|c|c|c|c|c|c|c|c|}
\hline \multicolumn{12}{|c|}{ CVT mesh with 8000 elements, $k=2$, dofs $=465721$} \\
\hline \multirow[t]{2}{*}{$p$} & \multirow[t]{2}{*}{$S_{p}^{\text {id }}$} & \multirow[t]{2}{*}{$T_{a s s}$} & \multirow[t]{2}{*}{$S_{p}$} & \multicolumn{2}{|c|}{ Mumps } & \multicolumn{3}{|c|}{ Block-Schur } & \multicolumn{3}{|c|}{ Block-Reg } \\
\hline & & & & $T_{\text {sol }}$ & $S_{p}$ & it & $T_{\text {sol }}$ & $S_{p}$ & it & $T_{\text {sol }}$ & $S_{p}$ \\
\hline 1 & - & 1688 & - & $\mathrm{F}$ & 2 & 607 & 398 & $x$ & 84 & 193 & 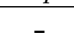 \\
\hline 4 & 4 & 497 & 3.4 & $\mathrm{~F}$ & - & 07 & 170 & 2.3 & 168 & 253 & 0.8 \\
\hline 8 & 8 & 2 & 5.9 & 2793 & - & 607 & 149 & 2.7 & 201 & 168 & 1.1 \\
\hline 16 & 16 & 156 & 10.8 & 2083 & 1.3 & 607 & 115 & 3.5 & 226 & 134 & 1.4 \\
\hline 32 & 32 & 83 & 20.3 & 1078 & 2.6 & 607 & 115 & 3.5 & 240 & 91 & 2.1 \\
\hline \multicolumn{12}{|c|}{ CVT mesh with 4000 elements, $\mathrm{k}=3$, dofs $=445951$} \\
\hline \multirow[t]{2}{*}{$p$} & \multirow[t]{2}{*}{$S_{p}^{\text {id }}$} & \multirow[t]{2}{*}{$T_{\text {ass }}$} & \multirow[t]{2}{*}{$S_{p}$} & \multicolumn{2}{|c|}{ Mumps } & \multicolumn{3}{|c|}{ Block-Schur } & \multicolumn{3}{|c|}{ Block-Reg } \\
\hline & & & & $T_{\text {sol }}$ & $S_{p}$ & it & $T_{\text {sol }}$ & $S_{p}$ & it & $T_{\text {sol }}$ & $S_{p}$ \\
\hline 4 & - & 2407 & - & 4335 & - & 3983 & 1168 & - & 2514 & 1882 & - \\
\hline 8 & 2 & 1276 & 1.9 & 2221 & 1.9 & 4386 & 1095 & 1.1 & 2706 & 1226 & 1.5 \\
\hline 16 & 4 & 779 & 3.1 & 1670 & 2.6 & 3901 & 831 & 1.4 & 2899 & 879 & 2.1 \\
\hline 32 & 8 & 414 & 5.8 & 830 & 5.2 & 4357 & 986 & 1.2 & 2849 & 543 & 3.5 \\
\hline
\end{tabular}

Table 3: Strong scaling test, CVT meshes. Same format as in Table 1

\begin{tabular}{c|c|c|c|cc|cc}
\hline \multicolumn{7}{|c}{ Cube mesh, $\mathbf{k}=\mathbf{2}, \mathbf{p}=\mathbf{8}$} \\
\hline$N_{P}$ & dofs & $T_{\text {ass }}$ & Mumps & \multicolumn{2}{|c}{ Block-Schur } & \multicolumn{2}{c}{ Block-Reg } \\
& & & $T_{\text {sol }}$ & it & $T_{\text {sol }}$ & it & $T_{\text {sol }}$ \\
\hline 512 & 19585 & 1 & 1 & 113 & 1 & 86 & 1 \\
4096 & 152065 & 13 & 14 & 78 & 9 & 123 & 9 \\
8000 & 295201 & 29 & 73 & 76 & 29 & 145 & 22 \\
13824 & 508033 & 53 & 270 & 72 & 77 & 168 & 48 \\
21952 & 804385 & 84 & 604 & 69 & 217 & 188 & 87 \\
\hline
\end{tabular}

Table 4: Optimality test, Cube meshes. $N_{P}=$ number of mesh polyhedra; dofs $=$ degrees of freedom; $T_{a s s}:=$ assembling time in seconds; $T_{\text {sol }}:=$ solution time in seconds; it:=GMRES iterations. The order of VEM discretization is fixed to $\mathrm{k}=2$. The runs are performed on 8 processors $(\mathrm{p}=8)$.

\begin{tabular}{c|c|c|c|cc|cc}
\hline \multicolumn{1}{c}{ Octa mesh, $\mathbf{k}=\mathbf{2}, \mathbf{p}=\mathbf{8}$} \\
\hline$N_{P}$ & dofs & $T_{\text {ass }}$ & Mumps & \multicolumn{2}{|c}{ Block-Schur } & \multicolumn{2}{c}{ Block-Reg } \\
& & & $T_{\text {sol }}$ & it & $T_{\text {sol }}$ & it & $T_{\text {sol }}$ \\
\hline 72 & 3121 & $<1$ & $<1$ & 367 & 2 & 88 & $<1$ \\
576 & 23809 & 3 & $<1$ & 498 & 4 & 140 & 2 \\
4608 & 185857 & 25 & 13 & 598 & 35 & 230 & 24 \\
9000 & 361201 & 60 & 66 & 559 & 102 & 277 & 58 \\
15552 & 622081 & 111 & 190 & 631 & 283 & 310 & 113 \\
\hline
\end{tabular}

Table 5: Optimality test, Octa meshes. Same format as in Table 4

[4] P. F. Antonietti, L. Mascotto, and M. Verani. A multigrid algorithm for the p-version of the virtual element method. ESAIM: Math. Model. Numer. Anal., 52(1):337-364, 2018. 

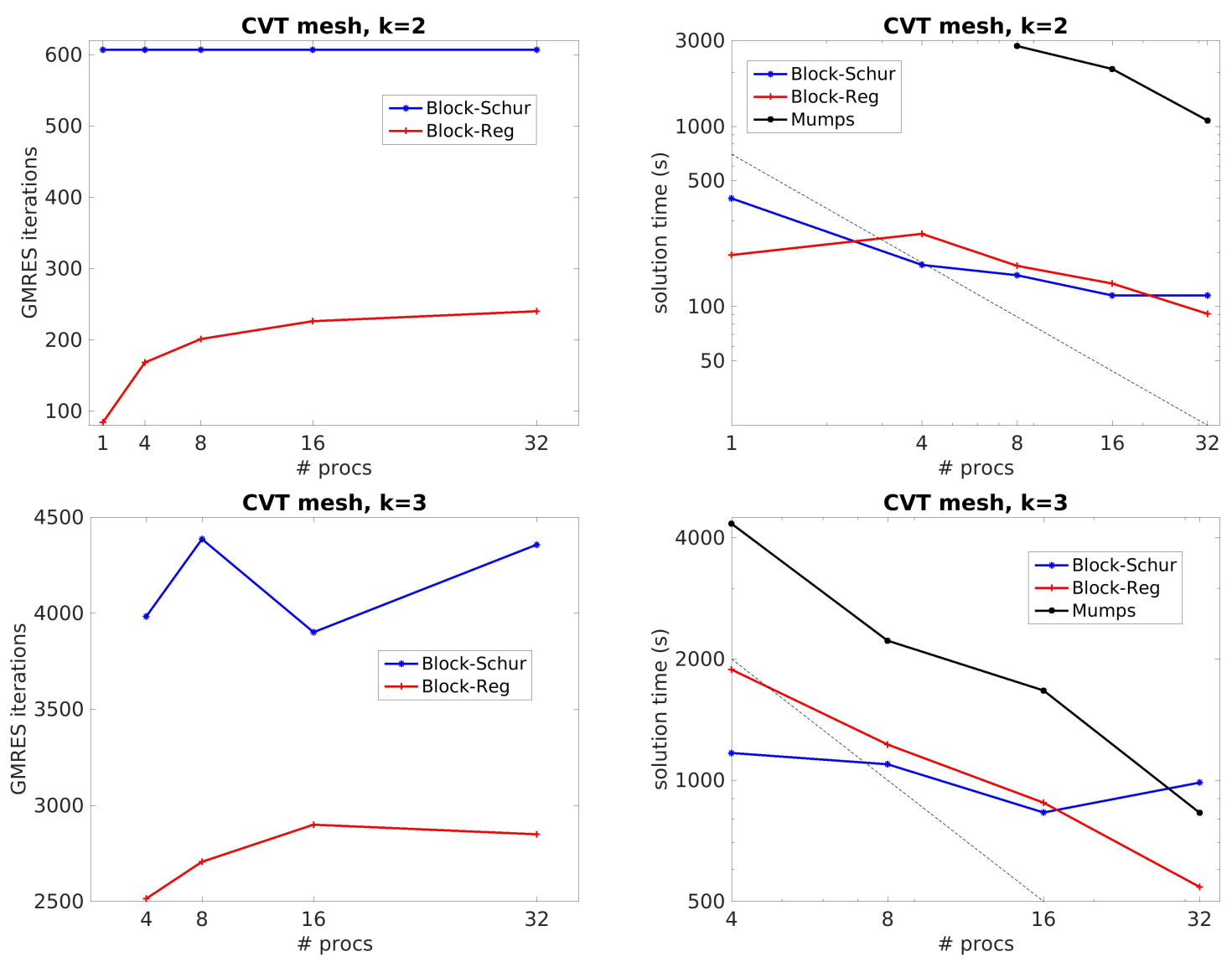

Figure 5: Strong scaling test, CVT meshes. Same format as in Fig. 3.

\begin{tabular}{c|c|c|c|cc|cc}
\hline \multicolumn{7}{c}{ CVT mesh, $\mathbf{k}=\mathbf{2}, \mathbf{p}=\mathbf{8}$} \\
\hline$N_{P}$ & dofs & $T_{\text {ass }}$ & Mumps & \multicolumn{2}{c}{ Block-Schur } & \multicolumn{2}{c}{ Block-Reg } \\
& & & $T_{\text {sol }}$ & it & $T_{\text {sol }}$ & it & $T_{\text {sol }}$ \\
\hline 27 & 1495 & 1 & $<1$ & 727 & 2 & 151 & $<1$ \\
125 & 6841 & 3 & $<1$ & 535 & 2 & 124 & 2 \\
1000 & 57019 & 33 & 20 & 652 & 10 & 142 & 16 \\
4000 & 231571 & 143 & 467 & 736 & 46 & 168 & 75 \\
8000 & 465721 & 285 & 2793 & 607 & 149 & 201 & 168 \\
\hline
\end{tabular}

Table 6: Optimality test, CVT meshes. Same format as in Table 4
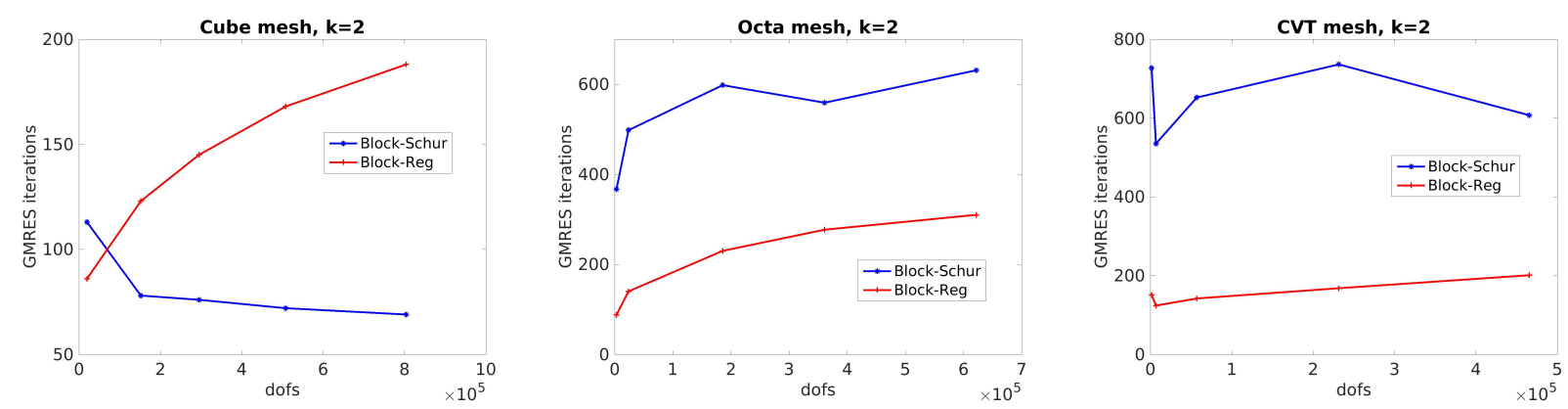

Figure 6: Optimality test. GMRES iterations as a function of the number of dofs for $k=2$ VEM discretization on Cube (left), Octa (center) and CVT (right) meshes.

[5] P. F. Antonietti, M. Sarti, and M. Verani. Multigrid algorithms for hp-discontinuous galerkin 
discretizations of elliptic problems. SIAM Journal on Numerical Analysis, 53(1):598-618, 2015.

[6] P. F. Antonietti, M. Verani, and L. Zikatanov. A two-level method for mimetic finite difference discretizations of elliptic problems. Computers $\mathcal{E}$ Mathematics with Applications, 70(11):2674 2687, 2015. Numerical Methods for Scientific Computations and Advanced Applications.

[7] O. Axelsson. Iterative Solution Methods. Cambridge University Press, 1994.

[8] O. Axelsson, R. Blaheta, P. Byczanski, J. Karátson, and B. Ahmad. Preconditioners for regularized saddle point problems with an application for heterogeneous Darcy flow problems. $J$. Comput. Appl. Math., 280:141-157, 2015.

[9] B. Ayuso de Dios, K. Lipnikov, and G. Manzini. The nonconforming virtual element method. ESAIM: Math. Model. Numer. Anal., 50(3):879-904, 2016.

[10] S. Balay, S. Abhyankar, M. F. Adams, J. Brown, P. Brune, K. Buschelman, L. Dalcin, V. Eijkhout, W. D. Gropp, D. Kaushik, M. G. Knepley, D. A. May, L. C. McInnes, R. T. Mills, T. Munson, K. Rupp, P. Sanan, B. F. Smith, S. Zampini, H. Zhang, and H. Zhang. PETSc Web page. http://www.mcs.anl.gov/petsc, 2018.

[11] S. Balay, S. Abhyankar, M. F. Adams, J. Brown, P. Brune, K. Buschelman, L. Dalcin, V. Eijkhout, W. D. Gropp, D. Kaushik, M. G. Knepley, D. A. May, L. C. McInnes, R. T. Mills, T. Munson, K. Rupp, P. Sanan, B. F. Smith, S. Zampini, H. Zhang, and H. Zhang. PETSc users manual. Technical Report ANL-95/11 - Revision 3.9, Argonne National Laboratory, 2018.

[12] S. Balay, W. D. Gropp, L. C. McInnes, and B. F. Smith. Efficient management of parallelism in object oriented numerical software libraries. In E. Arge, A. M. Bruaset, and H. P. Langtangen, editors, Modern Software Tools in Scientific Computing, pages 163-202. Birkhäuser Press, 1997.

[13] L. Beirão da Veiga, F. Brezzi, L. D. Marini, and A. Russo. The hitchhiker's guide to the virtual element method. Math. Models Methods Appl. Sci., 24(08):1541-1573, 2014.

[14] L. Beirão da Veiga, F. Dassi, and A. Russo. High-order virtual element method on polyhedral meshes. Comput. Math. Appl., 74(5):1110-1122, 2017.

[15] L. Beirão da Veiga, F. Brezzi, A. Cangiani, G. Manzini, L. D. Marini, and A. Russo. Basic principles of virtual element methods. Math. Mod. Meth. Appl. Sci., 23(1):199-214, 2013.

[16] L. Beirão da Veiga, F. Brezzi, F. Dassi, L. D. Marini, and A. Russo. A family of three-dimensional virtual elements with applications to magnetostatic. arXiv preprint arXiv:1804.10497, 2018.

[17] L. Beirão Da Veiga, F. Brezzi, F. Dassi, L. D. Marini, and A. Russo. Serendipity virtual elements for general elliptic equations in three dimensions. Chinese Annals of Mathematics, Series B, 39(2):315-334, Mar 2018.

[18] L. Beirão da Veiga, F. Brezzi, and L. D. Marini. Virtual elements for linear elasticity problems. SIAM J. Numer. Anal., 51(2):794-812, 2013.

[19] L. Beirão da Veiga, F. Brezzi, L. D. Marini, and A. Russo. H(div) and h(curl)-conforming virtual element methods. Numer. Math., 133:303-332, 2016.

[20] L. Beirão da Veiga, F. Brezzi, L. D. Marini, and A. Russo. Mixed virtual element methods for general second order elliptic problems on polygonal meshes. ESAIM: Math. Model. Numer. Anal., 50(3):727-747, 2016.

[21] L. Beirão da Veiga and A. Ern. Preface. ESAIM: Math. Model. Numer. Anal., 50(3):633-634, 2016.

[22] L. Beirão da Veiga, K. Lipnikov, and G. Manzini. The Mimetic Finite Difference Method for Elliptic Problems. Springer International Publishing, 2014. 
[23] L. Beirão da Veiga, C. Lovadina, and G. Vacca. Divergence free virtual elements for the stokes problem on polygonal meshes. ESAIM: Math. Model. Numer. Anal., 51(2):509-535, 2017.

[24] M. F. Benedetto, S. Berrone, S. Pieraccini, and S. Scialò. The virtual element method for discrete fracture network simulations. Comput. Meth. Appl. Mech. Eng., 280:135-156, 2014.

[25] M. Benzi, G. H. Golub, and J. Liesen. Numerical solution of saddle point problems. Acta numerica, 14:1-137, 2005.

[26] S. Bertoluzza, M. Pennacchio, and D. Prada. BDDC and FETI-DP for the virtual element method. Calcolo, 54(4):1565-1593, 2017.

[27] D. Boffi, F. Brezzi, and M. Fortin. Mixed finite element methods and applications, volume 44 of Springer Series in Computational Mathematics. Springer, Heidelberg, 2013.

[28] Susanne C Brenner and Li-Yeng Sung. Virtual element methods on meshes with small edges or faces. Mathematical Models and Methods in Applied Sciences, pages 1-46, 2018.

[29] F. Brezzi, R. S. Falk, and L. D. Marini. Basic principles of mixed virtual element methods. ESAIM: Math. Model. Numer. Anal., 48(4):1227-1240, 2014.

[30] F. Brezzi, K. Lipnikov, and M. Shashkov. Convergence of the mimetic finite difference method for diffusion problems on polyhedral meshes. SIAM Journal on Numerical Analysis, 43(5):1872-1896, 2005 .

[31] J. G. Calvo. On the approximation of a virtual coarse space for domain decomposition methods in two dimensions. Math. Mod. Meth. Appl. Sci., 28(07):1267-1289, 2018.

[32] A. Cangiani, G. Manzini, and O. J. Sutton. Conforming and nonconforming virtual element methods for elliptic problems. IMA J. Numer. Anal., 37(3):1317-1354, 2017.

[33] Lourenço Beirão da Veiga, David Mora, Gonzalo Rivera, and Rodolfo Rodríguez. A virtual element method for the acoustic vibration problem. Numerische Mathematik, 136(3):725-763, 2017.

[34] F. Dassi and L. Mascotto. Exploring high-order three dimensional virtual elements: Bases and stabilizations. Comput. Math. Appl., 75(9):3379-3401, 2018.

[35] Q. Du, V. Faber, and M. Gunzburger. Centroidal voronoi tessellations: Applications and algorithms. SIAM Rev., 41(4):637-676, 1999.

[36] Alessio Fumagalli and Eirik Keilegavlen. Dual virtual element method for discrete fractures networks. SIAM Journal on Scientific Computing, 40(1):B228-B258, 2018.

[37] A. L. Gain, C. Talischi, and G. H. Paulino. On the virtual element method for three-dimensional linear elasticity problems on arbitrary polyhedral meshes. Comput. Meth. Appl. Mech. Eng., 282:132-160, 2014.

[38] G. H. Golub and C. Greif. On solving block-structured indefinite linear systems. SIAM J. Sci. Comput., 24(6):2076-2092, 2003.

[39] A. Klawonn. Block-triangular preconditioners for saddle point problems with a penalty term. SIAM J. Sci. Comput., 19(1):172-184, 1998.

[40] K.-A. Mardal and R. Winther. Preconditioning discretizations of systems of partial differential equations. Numer. Lin. Alg. Appl., 18(1):1-40, 2011.

[41] L. Mascotto. Ill-conditioning in the virtual element method: Stabilizations and bases. Numer. Meth. Part. Diff. Eq., 34(4):1258-1281, 2018. 
[42] T. P. Mathew. Schwarz alternating and iterative refinement methods for mixed formulations of elliptic problems, part i: Algorithms and numerical results. Numer. Math., 65(1):445-468, 1993.

[43] T. P. Mathew. Schwarz alternating and iterative refinement methods for mixed formulations of elliptic problems, part II: Convergence theory. Numer. Math., 65(1):469-492, 1993.

[44] D.-S. Oh, O. B. Widlund, S. Zampini, and C. R. Dohrmann. BDDC algorithms with deluxe scaling and adaptive selection of primal constraints for raviart-thomas vector fields. Math. Comp., 87(310):659-692, 2017.

[45] C. H. Rycroft. Voro++: A three-dimensional voronoi cell library in c++. Chaos, 19(4):041111, 2009 .

[46] Y. Saad. Iterative Methods for Sparse Linear Systems. SIAM, 2003.

[47] V. Simoncini. Block triangular preconditioners for symmetric saddle-point problems. Appl. Numer. Math., 49(1):63-80, 2004.

[48] G. Vacca. Virtual element methods for hyperbolic problems on polygonal meshes. Comput. Math. Appl., 74(5):882-898, 2017.

[49] G. Vacca and L. Beirão da Veiga. Virtual element methods for parabolic problems on polygonal meshes. Numer. Meth. Part. Diff. Eq., 31(6):2110-2134, 2015.

[50] S. Zampini and X. Tu. Multilevel balancing domain decomposition by constraints deluxe algorithms with adaptive coarse spaces for flow in porous media. SIAM J. Sci. Comput., 39(4):A1389A1415, 2017.

[51] W. Zulehner. Analysis of iterative methods for saddle point problems: a unified approach. Math. Comp., 71(238):479-506, 2001. 